\title{
THE PRINCIPLES OF THE CONCEPTUAL ORGANIZATION AND CONSTRUCTION OF PATENT PROTECTION
}

\section{for smart integrative technical systems containing complex subsystems, managed and on-line controlled at the level of capabilities of quantum computers containing elements of artificial intelligence and artificial neural networks}

\author{
Yerzhan Kusparmakov \\ - Engineer, Inventor, Doctor of Philosophy (PhD) Major in Computer Science \\ - Professional Member of the New York Academy of Science \\ - Academician of the International Informatization Academy \\ - Chief Product Manager at SAMSUNG Electronics \\ e-mail: kusparmakov@gmail.com
}

\begin{abstract}
The integration of software solutions and mobile applications into innovative technical systems of all levels in the current process of transforming classical technologies into smart technologies and transforming the entire hierarchy of technical systems and their interconnections into smart technical systems is the basis for the formation of such innovative products that are most in demand on the markets.

As the practice of such innovative development shows, the use of methods and techniques of the inventive process adapted to today's elemental base and modern composite materials from generating an idea to the complete formation of materials for an application for an invention can be successfully carried out with the well-known Theory and Algorithm for solving inventive problems.

Of particular importance in the innovation process are relations with investors and potential strategic partners in the commercial application of the created innovative product.

Keywords: Patent, Licensing, Quantum Computers, Artificial Intelligence (AI), Artificial Neural Networks (ANN), Augmented Reality (AR), Smart technology
\end{abstract}

The integration of software solutions and mobile applications into innovative technical systems of all levels in the current process of transforming classical technologies into smart technologies and transforming the entire hierarchy of technical systems and their interconnections into smart technical systems is the basis for the formation of such innovative products that are most in demand on the markets.

As the practice of such innovative development shows, the use of methods and techniques of the inventive process adapted to today's elemental base and modern composite materials from generating an idea to the complete formation of materials for an application for an invention can be successfully carried out with the well-known Theory and Algorithm for solving inventive problems. 
The use of Theory of Inventive Problem Solving (TIPS) and Algorithms for Solving Inventive Problems (ASIP) is of course only one of many possible analytical tools in the preparation of a patent application.

The relations with investors and potential strategic partners in the commercial application of the created innovative product are of particular importance in the innovation process.

To convince the investor that investments in the proposed project will bring financial success, it is necessary to explain in detail the essence of the project and to reveal all technological and innovative secrets to the experts.

It is impossible without serious protection of the engineering solutions that form the basis of the innovative product at all stages of the project development.

Non-traditional types and forms of collective investment have now appeared and are successfully developing, and this also motivates the project authors to constantly search for more and more reliable forms of protection of their inventions.

Provisional Patent Application is an inexpensive and reliable protection option for up to 1 year.

The use of TIPS and ASIP, as already mentioned above, with the necessary and sufficient updating of the basic techniques and methods, is only one of the many possible tools in the preparation of the patent application and in many cases requires system modification and optimization taking into account new innovative circumstances.

For each specific case, taking into account all circumstances, reasons, objectives and conditions, and taking into account the results of patent forecasting, in full compliance with the current US Patent Law (America Invents Act), an applicant company or an individual inventor may have, and most often need, an integrative program in which the original system and structure of the patent application are precisely developed.

A patent licensing strategy, including a consistent patent forecasting process, can also be, and most often should be, developed for each individual applicant or inventor.

This manual is also fully suitable for compiling application materials for filing (Non Provisional Patent Application ..., - Utility Patent Application).

\section{APPARATUS AND METHOD FOR ...}

The title of the proposed invention should contain not only a brief definition of the nature of the engineering solution, but also an extremely brief commercial description of its impact on the market within the scope of the proposed engineering solution. The title should not contain any advertising characteristics..., - such as effective, best, etc. 
In the title of the invention, it is very important to take into account the market conditions existing at the time of application filing. If the purpose of the authors (or their companies and financial partners) and applicants is to further attract additional investment in the development of the project, the basis of which should be the claimed engineering solution, it is preferable to consider in the title the known technological and commercial interests of potential partners.

Since the proof of non-obviousness of the claimed engineering solution is very important for a patent expert in the United States, it is extremely important to take into account the criteria and characterization of non-obviousness in the title of the invention.

Technical systems related to the so-called smart technologies and equipment, as a rule, should include the software part, which in itself is not a technical solution and cannot be classified as a technical system.

But since software solutions are the basis of the control and monitoring systems of any modern smart technology, this should be taken into account in the name of the invention, that is, the name of the invention should be presented in the form: - Device, program, system and method; or - An apparatus, program, system and method.

In addition, when classifying a technical system as a smart system having elements of artificial intelligence and artificial neural networks, it is also necessary to have information storages with the corresponding amount of information and corresponding speed.

This factor should be reflected in the name, for example ..., - The device, program, system with memory drives, and the associated method of use.

\section{REFERENCE TO RELATED APPLICATIONS}

[0001] This application claims the priority benefit under 35 U.S.C. $\$ 119($ e) of U.S. Provisional Application Nos. 61/, filed...; and 61/, filed... The entire disclosures of each of these applications are incorporated herein by reference..., - this section contains information on all provisional patent applications that have been filed by the authors of the invention or the applicant prior to the execution and filing of this application.

If the provisional patent applications in the United States (Provisional Patent Applications) have not been filed, this section of the application should specify information on all previous applications on this topic and this technical field, which were filed by the authors of the invention or by the applicant (in the name of the authors of this application or other authors) before the execution and filing of this application.

\section{TECHNICAL FIELD}


[0002] The invention relates to apparatus and methods for..., - this section describes the field of methods and technology to which the claimed engineering solution relates.

The nature of belonging to one or another technical field should be clearly reasoned and related to the distinctive features of the claimed engineering solution by cause-effect relations characterized and formulated understandable for a semi-skilled specialist in this field.

If the distinctive features of the claimed engineering solution are obvious for one field and not obvious for another, it is necessary to stop at this and try to give an analytical explanation of this phenomenon.

It is especially important to characterize the problems and contradictions peculiar to and obvious for the technical field selected for the invention and for the development of this thesis for the field of methods and technology to which the claimed engineering solution relates.

Due to the fact that, unlike the European Patent Office, in the United States, under certain conditions, a patent can be granted for a computer program, it is preferable, if possible, to have an integration solution for the claimed engineering solution in the form of..., - Program, system, method and device for their application.

\section{DESCRIPTION OF THE RELATED TECHNOLOGY}

[0003] - this section consists of two parts. The first part provides a more specified, definite, local analytical assessment of the field of methods and technology, to which the claimed engineering solution relates. The most characteristic features of the technology, its known disadvantages and advantages are described.

[0004] - in this part of the section, it is preferable to start the search and preliminary analysis of the contradictions of a general nature, which are known and characteristic of the technology to which the inventors relate it.

\section{BACKGROUND OF THE INVENTION}

[0005] - this section, which is one of the most important in the application, provides an indepth analysis of patent search results for all options and sources of published patent information, at least for the US Patent Office, and better if for the European Patent Office, and even better if you give a comparative analysis of known engineering solutions and, in conclusion, bring the results of structural element-wise analysis. 
After that, using the TIPS and ASIP tools, it is necessary to search, formulate and comparatively analyze all kinds of contradictions in the known engineering solutions and determine and characterize a possible ideal final result.

Then it is necessary to analyze the possibility of the fundamental achievement of the ideal final result in the claimed engineering solution by using equipment, technology, materials and means of control and management, known at the time of application filing.

\section{SUMMARY}

[0006] According to some embodiments of the invention..., - this section defines the first local ultimate objective of the invention and defines the way to achieve it within the framework of compliance with the claims; provides identification of the local objective and determines whether it is enough to achieve the objective in view in order to state the achievement of at least a partial or local ideal final result.

[0007] In some embodiments, programs, systems and methods of..., - this section defines the second local ultimate objective of the invention and defines the way to achieve it within the framework of compliance with the claims; provides identification of the local objective and determines whether it is enough to achieve the objective in view in order to state the achievement of at least a partial or local ideal final result.

[0008] In some embodiments, further programs, systems and methods for..., - this section defines the third local ultimate objective of the invention and defines the way to achieve it within the framework of compliance with the claims; provides identification of the local objective and determines whether it is enough to achieve the objective in view in order to state the achievement of at least a partial or local ideal final result.

[0009] Also in some further embodiments, programs, systems and methods of...,- this section defines the first integrated local ultimate objective of the invention and defines the way to achieve it within the framework of compliance with the claims; provides identification of the local objective and determines whether it is enough to achieve the objective in view in order to state the achievement of at least a partial or local ideal final result.

[0010] In some embodiments, an apparatus is provided with..., - this section defines the first local ultimate objective of the invention related cause-effect with the second local ultimate objective of the invention and defines the way to achieve the specified objective integration within the framework of compliance with the claims; provides identification of the local integrated objective and determines whether it is enough to achieve this objective in view in order to state the achievement of at least a partial or local ideal final result. 
[0011] - this section defines the first local ultimate objective of the invention related causeeffect with the second and third local ultimate objectives of the invention and defines the way to achieve this objective integration within the framework of compliance with the claims; provides identification of the local integrated objective and determines whether it is enough to achieve this objective in view in order to state the achievement of at least a partial or local ideal final result.

[0012] In some embodiments, an apparatus for..., - this section defines the first local ultimate objective of the invention in terms of solutions for the apparatus related cause-effect with the second local ultimate objective of the invention also related to the apparatus and defines the way to achieve the specified objective integration within the framework of compliance with the claims; provides identification of the local integrated objective and determines whether it is enough to achieve this objective in view in order to state the achievement of at least a partial or local ideal final result.

[0013] In some other embodiments, an apparatus is provided with a system for..., - in this section, one of the known systems, that is necessary for its declared duty cycle, is added to the apparatus and the presence or absence of a jump effect is analyzed.

[0014] In some other embodiments, an apparatus is provided with a system for..., - in this section, the second of the known systems, that is necessary for its declared duty cycle, is added to the apparatus and the presence or absence of a jump effect is analyzed.

[0015] In some embodiments of the various apparatus, the..., - in this section, a combination of the known systems integrated with the apparatus into an integrative system, that is necessary for its declared duty cycle, is added to the apparatus and the presence or absence of a jump effect is analyzed.

[0016] - in this section, a combination of the known systems, that is necessary for its declared duty cycle, is added to the apparatus one by one, and the presence or absence of a jump effect is analyzed.

[0017] The system for..., - in this section, a structural analysis of the system (s) of the engineering solution is carried out and the sufficiency of the presence of the combination of all necessary systems in the engineering solution to ensure the achievement of an ideal final result is monitored.

[0018] Structurally, the apparatus can be provided with a...,- in this section, a structural analysis of the nodes and components of the apparatus is carried out.

[0019] In the apparatus, the system for..., - this section shows how design features affect the achievement of an ideal final result. 
[0020] The apparatus can also comprise a system for..., - this section considers the possibility of including various additional functionally independent and autonomous systems into the apparatus that can enhance the effect of the use of the proposed invention and thereby ensure a more confident achievement of the ideal final result.

[0021] In some embodiments, the method of operation of the device involves..., - this section analyzes local and integral objectives in dynamics or action, analyzes methods of achieving the objectives, and compares parameters after achieving a specified objective with an ideal final result.

[0022] An external..., - this section considers additional possible aspects of improving and modifying objectives and ways to achieve them, as well as analyzes and assesses the potential for achieving additional components of an ideal final result.

[0023] The following can be provided to..., - in this section, various actions and constructive improvements are considered that can complement the essence of the ideal final result with new content.

[0024] In some embodiments..., - this section gives the final definition of the fully integrated local ultimate objective of the invention and defines the way to achieve it within the framework of compliance with the claims.

The identification of the local and fully integrated objective in view is given and it is determined whether it is enough to achieve this objective in order to state the achievement of at least a partial or local ideal final result.

\section{BRIEF DESCRIPTION OF THE DRAWINGS}

[0025] The invention will be better understood from the Detailed Description and from the appended drawings, which are meant to illustrate and not to limit the invention. The Figures are not necessarily drawn to scale, nor are the relative sizes of parts within the Figures necessary in proportion to one another, - the invention will be better understood from the detailed description and from the appended drawings, which are meant to illustrate and not to limit the invention. The Figures are not necessarily drawn to scale, nor are the relative sizes of parts within the Figures necessary in proportion to one another.

[0026] Figure 1 is an example of a..., - this figure represents a general schematic flowchart of an engineering system/supersystem, in which all subsystems and their communication with each other as well as outputs and lines of communication with the supersystem are displayed with a focus on the functional features and differences integrated into the supersystem of software elements in combination with the management and control systems of the supersystem comprising elements of artificial intelligence and artificial neural networks. 
[0027] Figure 2 is an example of a ...,- this figure represents a general schematic flowchart of a leading and central engineering system/subsystem, in which all functionally related subsystems and their communication with each other as well as outputs and lines of communication with the supersystem are displayed with a focus on the functional features and differences integrated into the supersystem and, if possible, subsystems of software elements, in combination with the management and control systems of the supersystem comprising elements of artificial intelligence and artificial neural networks.

Technical details and software functions, as well as algorithms characterizing the nonobviousness of these solutions in combination with the general principles of use of elements of artificial intelligence and artificial neural networks are also reflected.

[0028] Figure 3 shows an example..., - this figure represents a general schematic flowchart of an auxiliary or local first engineering system/subsystem, in which all functionally related subsystems and their communication with each other as well as outputs and lines of communication with the supersystem are displayed with a focus on the functional features and differences integrated into the supersystem and, if possible, subsystems of software elements, in combination with the management and control systems of the supersystem comprising elements of artificial intelligence and artificial neural networks.

Technical details and software functions, as well as algorithms characterizing the nonobviousness of these solutions in combination with the general principles of use of elements of artificial intelligence and artificial neural networks are also reflected.

[0029] Figure 4 shows an example of components of the..., - this figure represents a general schematic flowchart of an auxiliary or local second engineering system/subsystem, in which all functionally related subsystems and their communication with each other as well as outputs and lines of communication with the supersystem are displayed with a focus on the functional features and differences integrated into the supersystem and, if possible, subsystems of software elements, in combination with the management and control systems of the supersystem comprising elements of artificial intelligence and artificial neural networks.

Technical details and software functions, as well as algorithms characterizing the nonobviousness of these solutions in combination with the general principles of use of elements of artificial intelligence and artificial neural networks are also reflected.

[0030] Figure 5 shows an example of a..., - this figure represents a general schematic flowchart of an auxiliary or local third engineering system/subsystem, in which all functionally related subsystems and their communication with each other as well as outputs and lines of communication with the supersystem are displayed with a focus on the functional features and differences integrated into the supersystem and, if possible, subsystems of software elements, in combination with the management and control systems of the supersystem comprising elements of artificial intelligence and artificial neural networks. 
Technical details and software functions, as well as algorithms characterizing the nonobviousness of these solutions in combination with the general principles of use of elements of artificial intelligence and artificial neural networks are also reflected.

[0031] Figure 6 is a..., - the illustrations to Figure 1 made as dependent graphic structures to dependent claims relating to independent claim 1.

[0032] Figure 7 shows an example of a..., - the illustrations to Figure 2 made as dependent graphic structures to dependent claims relating to independent claim 2.

[0033] Figure 8 is an example of a..., - the illustrations to Figure 3 made as dependent graphic structures to dependent claims relating to independent claim 3.

[0034] Figure 9 is an example of a...,- the illustrations to Figure 4 made as dependent graphic structures to dependent claims relating to independent claim 4.

[0035] Figure 10 is an example of a..., - the illustrations to Figure 5 made as dependent graphic structures to dependent claims relating to independent claim 5.

[0036] Figure 11 is an example of a...,- this figure represents a general schematic flowchart or interaction algorithm of an auxiliary or local first engineering system/subsystem, in which all functionally related subsystems and their communication with each other as well as outputs and lines of communication with the supersystem are displayed with a focus on the functional features and differences integrated into the supersystem and, if possible, subsystems of software elements, in combination with the management and control systems of the supersystem comprising elements of artificial intelligence and artificial neural networks.

Technical details and software functions, as well as algorithms characterizing the nonobviousness of these solutions in combination with the general principles of use of elements of artificial intelligence and artificial neural networks are also reflected.

[0037] Figure 12 is an example of an..., - this figure represents a general schematic flowchart or interaction algorithm of an auxiliary or local second, in order of importance, engineering system/subsystem, in which all functionally related subsystems and their communication with each other as well as outputs and lines of communication with the supersystem are displayed with a focus on the functional features and differences integrated into the supersystem and, if possible, subsystems of software elements, in combination with the management and control systems of the supersystem comprising elements of artificial intelligence and artificial neural networks.

Technical details and software functions, as well as algorithms characterizing the nonobviousness of these solutions in combination with the general principles of use of elements of artificial intelligence and artificial neural networks are also reflected. 
[0038] Figure 13 is an example of a..., - this figure represents a general schematic flowchart or interaction algorithm of an auxiliary or local third, in order of importance, engineering system/subsystem, in which all functionally related subsystems and their communication with each other as well as outputs and lines of communication with the supersystem are displayed with a focus on the functional features and differences integrated into the supersystem and, if possible, subsystems of software elements, in combination with the management and control systems of the supersystem comprising elements of artificial intelligence and artificial neural networks.

Technical details and software functions, as well as algorithms characterizing the nonobviousness of these solutions in combination with the general principles of use of elements of artificial intelligence and artificial neural networks are also reflected.

[0039] Figure 14 is an example of a..., - this figure represents a general schematic flowchart or interaction algorithm of an auxiliary or local fourth, in order of importance, engineering system/subsystem, in which all functionally related subsystems and their communication with each other as well as outputs and lines of communication with the supersystem are displayed with a focus on the functional features and differences integrated into the supersystem and, if possible, subsystems of software elements, in combination with the management and control systems of the supersystem comprising elements of artificial intelligence and artificial neural networks.

Technical details and software functions, as well as algorithms characterizing the nonobviousness of these solutions in combination with the general principles of use of elements of artificial intelligence and artificial neural networks are also reflected.

[0040] Figure 15 is an example of a..., - this figure represents a general schematic flowchart or interaction algorithm of an auxiliary or local fifth, in order of importance, engineering system/subsystem, in which all functionally related subsystems and their communication with each other as well as outputs and lines of communication with the supersystem are displayed with a focus on the functional features and differences integrated into the supersystem and, if possible, subsystems of software elements, in combination with the management and control systems of the supersystem comprising elements of artificial intelligence and artificial neural networks.

Technical details and software functions, as well as algorithms characterizing the nonobviousness of these solutions in combination with the general principles of use of elements of artificial intelligence and artificial neural networks are also reflected.

[0041] Figure 16 is an example of a..., - the illustrations to Figure 11 made as dependent graphic structures to dependent claims relating to independent claim 11. 
[0042] Figure 17 shows an example of..., - the illustrations to Figure 12 made as dependent graphic structures to dependent claims relating to independent claim 12.

[0043] Figure 18 is an example of a..., - the illustrations to Figure 13 made as dependent graphic structures to dependent claims relating to independent claim 13.

[0044] Figure 19 is an example of a....., - the illustrations to Figure 14 made as dependent graphic structures to dependent claims relating to independent claim 14.

[0045] Figure 20 is an example of a..., - the illustrations to Figure 15 made as dependent graphic structures to dependent claims relating to independent claim 15.

[0046] Figure 21 is an example of a..., - this figure represents a general schematic flowchart or algorithm of complex integration of an auxiliary or local first, in order of importance, engineering system/subsystem into a central supersystem, and all functionally related subsystems and their communication with each other as well as outputs and lines of communication with the supersystem are displayed in such engineering system/subsystem, with a focus on the functional features and differences integrated into the supersystem and, if possible, subsystems of software elements, in combination with the management and control systems of the supersystem comprising elements of artificial intelligence and artificial neural networks.

Technical details and software functions, as well as algorithms characterizing the nonobviousness of these solutions in combination with the general principles of use of elements of artificial intelligence and artificial neural networks are also reflected.

[0047] Figure 22 is an example of a..., - this figure represents a general schematic flowchart or algorithm of complex integration of an auxiliary or local second, in order of importance, engineering system/subsystem into a central supersystem, and all functionally related subsystems and their communication with each other as well as outputs and lines of communication with the supersystem are displayed in such engineering system/subsystem, with a focus on the functional features and differences integrated into the supersystem and, if possible, subsystems of software elements, in combination with the management and control systems of the supersystem comprising elements of artificial intelligence and artificial neural networks.

Technical details and software functions, as well as algorithms characterizing the nonobviousness of these solutions in combination with the general principles of use of elements of artificial intelligence and artificial neural networks are also reflected.

[0048] Figure 23 is an example of a..., - this figure represents a general schematic flowchart or algorithm of complex integration of an auxiliary or local third, in order of importance, engineering system/subsystem into a central supersystem, and all functionally related 
subsystems and their communication with each other as well as outputs and lines of communication with the supersystem are displayed in such engineering system/subsystem, with a focus on the functional features and differences integrated into the supersystem and, if possible, subsystems of software elements, in combination with the management and control systems of the supersystem comprising elements of artificial intelligence and artificial neural networks.

Technical details and software functions, as well as algorithms characterizing the nonobviousness of these solutions in combination with the general principles of use of elements of artificial intelligence and artificial neural networks are also reflected.

[0049] Figure 24 is an example of a..., - this figure represents a general schematic flowchart or algorithm of complex integration of an auxiliary or local fourth, in order of importance, engineering system/subsystem into a central supersystem, and all functionally related subsystems and their communication with each other as well as outputs and lines of communication with the supersystem are displayed in such engineering system/subsystem, with a focus on the functional features and differences integrated into the supersystem and, if possible, subsystems of software elements, in combination with the management and control systems of the supersystem comprising elements of artificial intelligence and artificial neural networks.

Technical details and software functions, as well as algorithms characterizing the nonobviousness of these solutions in combination with the general principles of use of elements of artificial intelligence and artificial neural networks are also reflected.

\section{DETAILED DESCRIPTION}

[0050] According to some embodiments, an..., - this section describes the elements of essential novelty contained in the proposed invention at the level of the basic engineering solution of the top position in the hierarchy of new or updated engineering solutions and their integrative combinations.

[0051] In some embodiments, a..., - this section describes the principles of integration of software solutions into the basic engineering solution.

[0052] In order to develop..., - this section describes the fundamental elements of development of all types of engineering solutions at the level of the basic engineering solution without integration with software and test solutions.

[0053] To develop a..., - this section describes methods and processes, as well as algorithms designed for deep integration of software solutions of all kinds as a whole and hierarchy of the basic engineering solution and its integrative combinations with engineering subsystems. 
[0054] It will be appreciated that some..., - this section shows the reference and consequence of the input of software solutions in the principal characteristic and connections in all interconnected and mutually integrated engineering systems, taking into account the essential elements of artificial intelligence and related artificial neural networks.

[0055] In some embodiments, the..., - this section describes characteristics and new distinctive features that appeared during integration of software solutions of all levels into the basic engineering solution.

[0056] It will be appreciated that the..., - this section shows the elements of essential novelty that appeared during integration of software solutions into classic-type engineering solutions of all levels of the hierarchy.

[0057] In some embodiments, the..., - this section details characteristics and working relations of artificial intelligence elements with the basic scheme of operation of engineering systems of all levels of the hierarchy with reference of the principles of essential novelty to the algorithm or principles of operation of the entire complex invented engineering system.

[0058] In some embodiments, an..., - this section details characteristics and working relations of elements of artificial intelligence and artificial neural networks with the basic scheme of operation of engineering systems of all levels of the hierarchy with reference of the principles of essential novelty to the algorithm or principles of operation of the entire complex invented engineering system.

[0059] Reference will now be made to the figures, in which like numerals refer to like parts throughout.

[0060] In Figure 1..., - this section provides a detailed description of Figure 1 with a legend of all reference characters and reference numerals or digital designations. All digital designations for this figure should be of the form - 101, 102... The following reference numerals identify the following features.

[0061] Figure 2 shows an example of a..., - this section provides a detailed description of Figure 2 with a legend of all reference characters and reference numerals or digital designations. All digital designations for this figure should be of the form - 201, 202... The following reference numerals identify the following features.

[0062] Figure 3 shows a..., - this section provides a detailed description of Figure 3 with a legend of all reference characters and reference numerals or digital designations. All digital designations for this figure should be of the form - 301, 302... The following reference numerals identify the following features.

[0063] Figure 4 shows a..., - this section provides a detailed description of Figure 4 with a legend of all reference characters and reference numerals or digital designations. All digital 
designations for this figure should be of the form - 401, 402... The following reference numerals identify the following features.

[0064] Figure 5 shows a..., - this section provides a detailed description of Figure 5 with a legend of all reference characters and reference numerals or digital designations. All digital designations for this figure should be of the form - 501, 502... The following reference numerals identify the following features.

[0065] Figure 6 shows a..., - this section provides a detailed description of Figure 6 with a legend of all reference characters and reference numerals or digital designations. All digital designations for this figure should be of the form - 601, 602... The following reference numerals identify the following features.

[0066] Figure 7 shows..., - this section provides a detailed description of Figure 7 with a legend of all reference characters and reference numerals or digital designations. All digital designations for this figure should be of the form - 701, 702... The following reference numerals identify the following features.

[0067] Figure 8 shows an..., - this section provides a detailed description of Figure 8 with a legend of all reference characters and reference numerals or digital designations. All digital

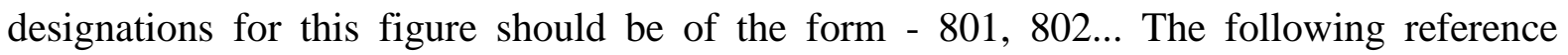
numerals identify the following features.

[0068] Figure 9 shows a..., - this section provides a detailed description of Figure 9 with a legend of all reference characters and reference numerals or digital designations. All digital designations for this figure should be of the form - 901, 902... The following reference numerals identify the following features.

[0069] Figure 10 shows a..., - this section provides a detailed description of Figure 10 with a legend of all reference characters and reference numerals or digital designations. All digital designations for this figure should be of the form - 1001, 1002... The following reference numerals identify the following features.

[0070] Figure 11 shows..., - this section provides a detailed description of Figure 11 with a legend of all reference characters and reference numerals or digital designations. All digital designations for this figure should be of the form $-1001,1002 \ldots$ The following reference numerals identify the following features.

[0071] Figure 12 shows ..., - this section provides a detailed description of Figure 12 with a legend of all reference characters and reference numerals or digital designations. All digital designations for this figure should be of the form - 1201, 1202... The following reference numerals identify the following features. 
[0072] Figure 13 shows a..., - this section provides a detailed description of Figure 13 with a legend of all reference characters and reference numerals or digital designations. All digital designations for this figure should be of the form - 1301, 1302... The following reference numerals identify the following features.

[0073] Figure 14 shows..., - this section provides a detailed description of Figure 14 with a legend of all reference characters and reference numerals or digital designations. All digital designations for this figure should be of the form - 1401, 1402... The following reference numerals identify the following features.

[0074] Figure 15 shows a..., - this section provides a detailed description of Figure 15 with a legend of all reference characters and reference numerals or digital designations. All digital designations for this figure should be of the form $-1501,1502 \ldots$ The following reference numerals identify the following features.

[0075] Figure 16 shows a..., - this section provides a detailed description of Figure 16 with a legend of all reference characters and reference numerals or digital designations. All digital designations for this figure should be of the form - 1601, 1602... The following reference numerals identify the following features.

[0076] Figure 17 shows..., - this section provides a detailed description of Figure 17 with a legend of all reference characters and reference numerals or digital designations. All digital designations for this figure should be of the form - 1701, 1702... The following reference numerals identify the following features.

[0077] Figure 18 shows a..., - this section provides a detailed description of Figure 18 with a legend of all reference characters and reference numerals or digital designations. All digital designations for this figure should be of the form - 1801, 1802... The following reference numerals identify the following features.

[0078] Figure 19 shows a..., - this section provides a detailed description of Figure 19 with a legend of all reference characters and reference numerals or digital designations. All digital designations for this figure should be of the form - 1901, 1902... The following reference numerals identify the following features.

[0079] Figure 20 shows..., - this section provides a detailed description of Figure 20 with a legend of all reference characters and reference numerals or digital designations. All digital designations for this figure should be of the form - 2001, 2002... The following reference numerals identify the following features.

[0080] Figure 21 shows a..., - this section provides a detailed description of Figure 21 with a legend of all reference characters and reference numerals or digital designations. All digital 
designations for this figure should be of the form - 2101, 2102... The following reference numerals identify the following features.

[0081] Figure 22 shows an..., - this section provides a detailed description of Figure 22 with a legend of all reference characters and reference numerals or digital designations. All digital designations for this figure should be of the form - 2201, 2202... The following reference numerals identify the following features.

[0082] Figure 23 shows a..., - this section provides a detailed description of Figure 23 with a legend of all reference characters and reference numerals or digital designations. All digital designations for this figure should be of the form - 2301, 2302... The following reference numerals identify the following features.

[0083] Figure 24 shows a..., - this section provides a detailed description of Figure 24 with a legend of all reference characters and reference numerals or digital designations.

\section{TEST RESULTS}

[0084]This section demonstrates the test results in the form of indicators, tables, graphs, and charts.

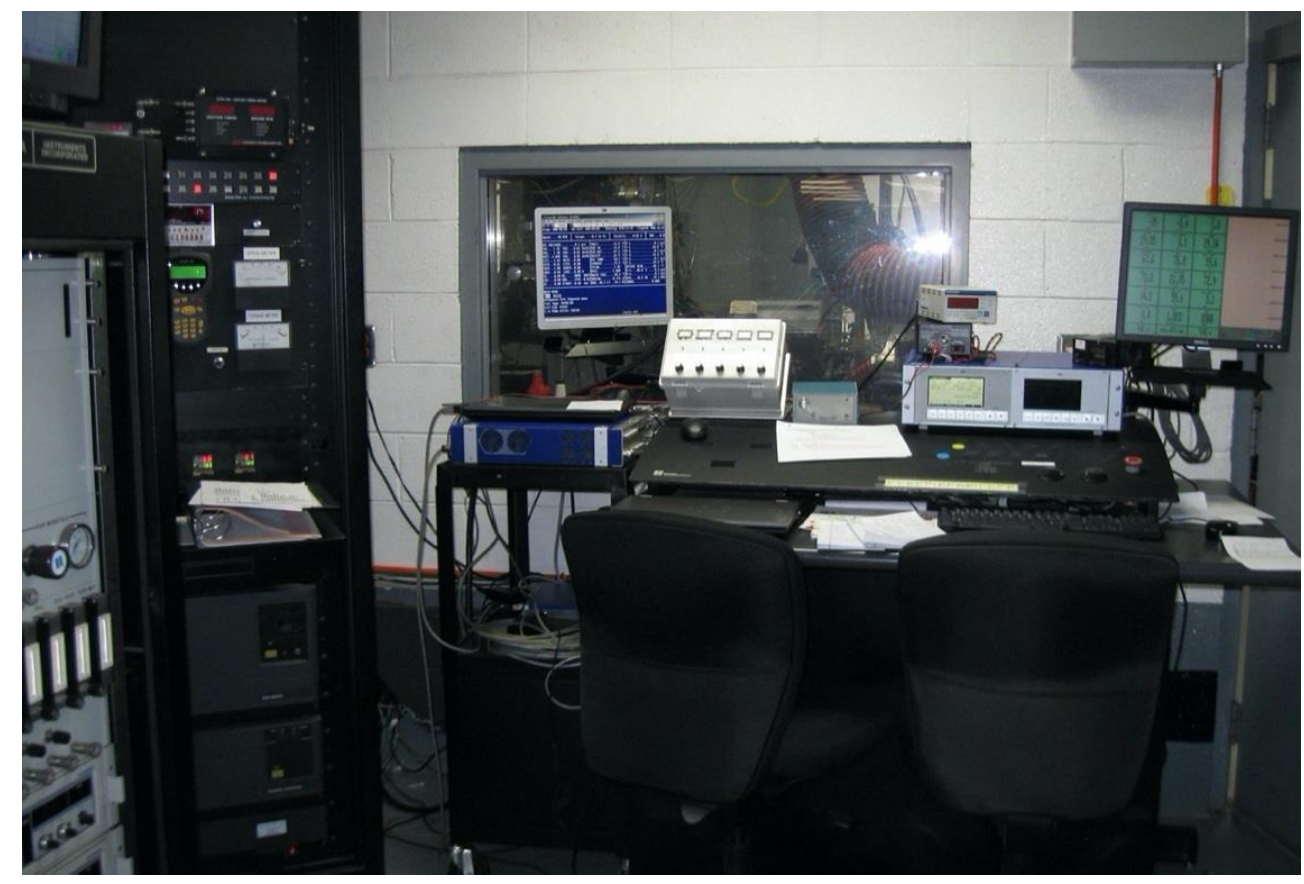

Figure 1, - the figure shows an example of the location of the system of analytical and control equipment of the test bench, intended for testing and analytical evaluation of a diesel engine. Bench systems include elements of artificial intelligence and artificial neural networks. It is very important to show and reveal the novelty of using this system to achieve an ideal end result or its equivalent in relation to the claimed material 


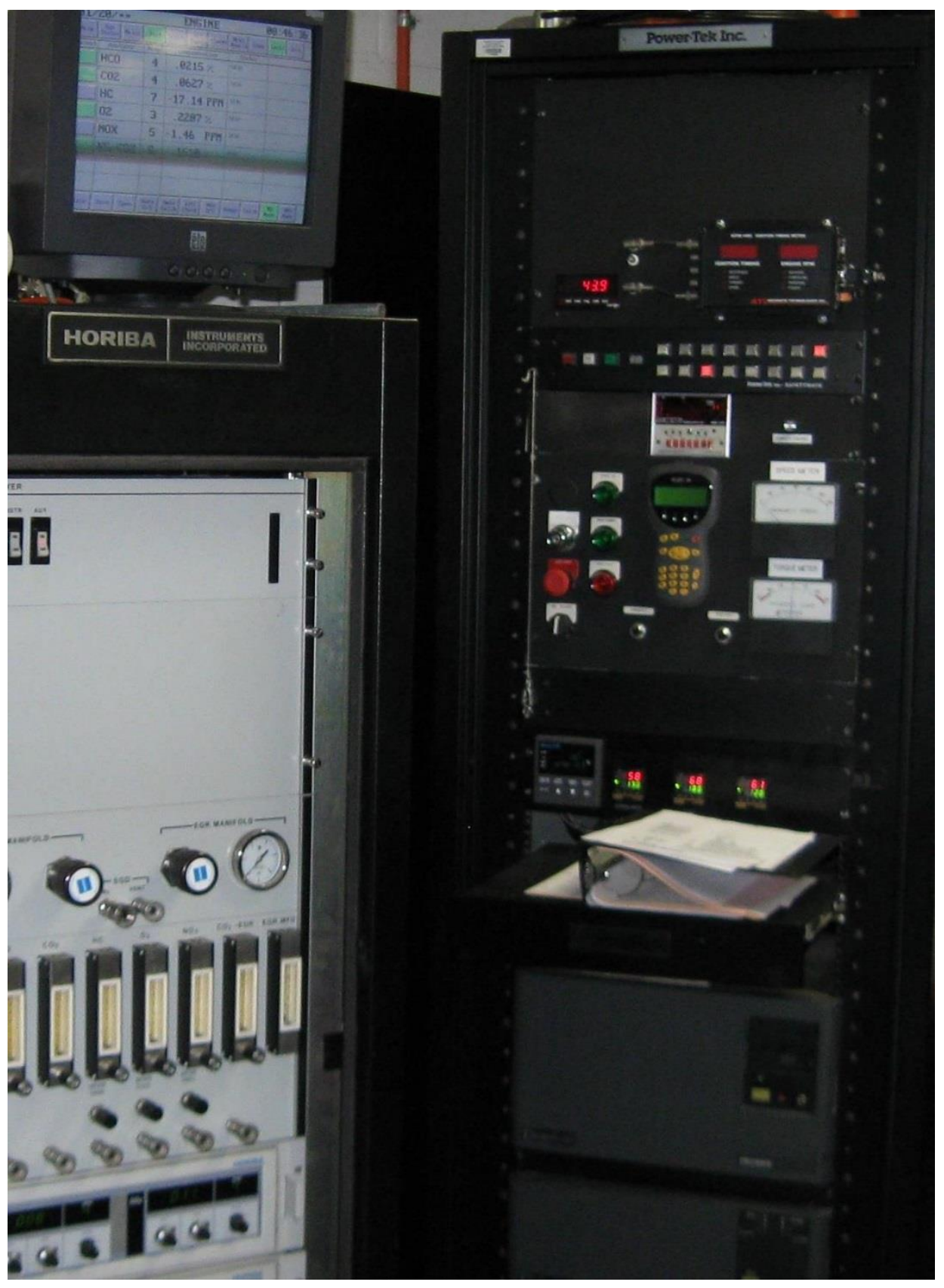

Figure 2, - the figure shows an example of a fragment of the location of the system of analytical and control equipment of the test bench, intended for testing and analytical evaluation of a diesel engine. Bench systems include elements of artificial intelligence and artificial neural networks. It is very important to show and disclose the novelty of using this system to achieve an ideal end result or its equivalent in relation to the claimed material, as well as to show the interaction of the control and analytical components of the system with elements of artificial intelligence 


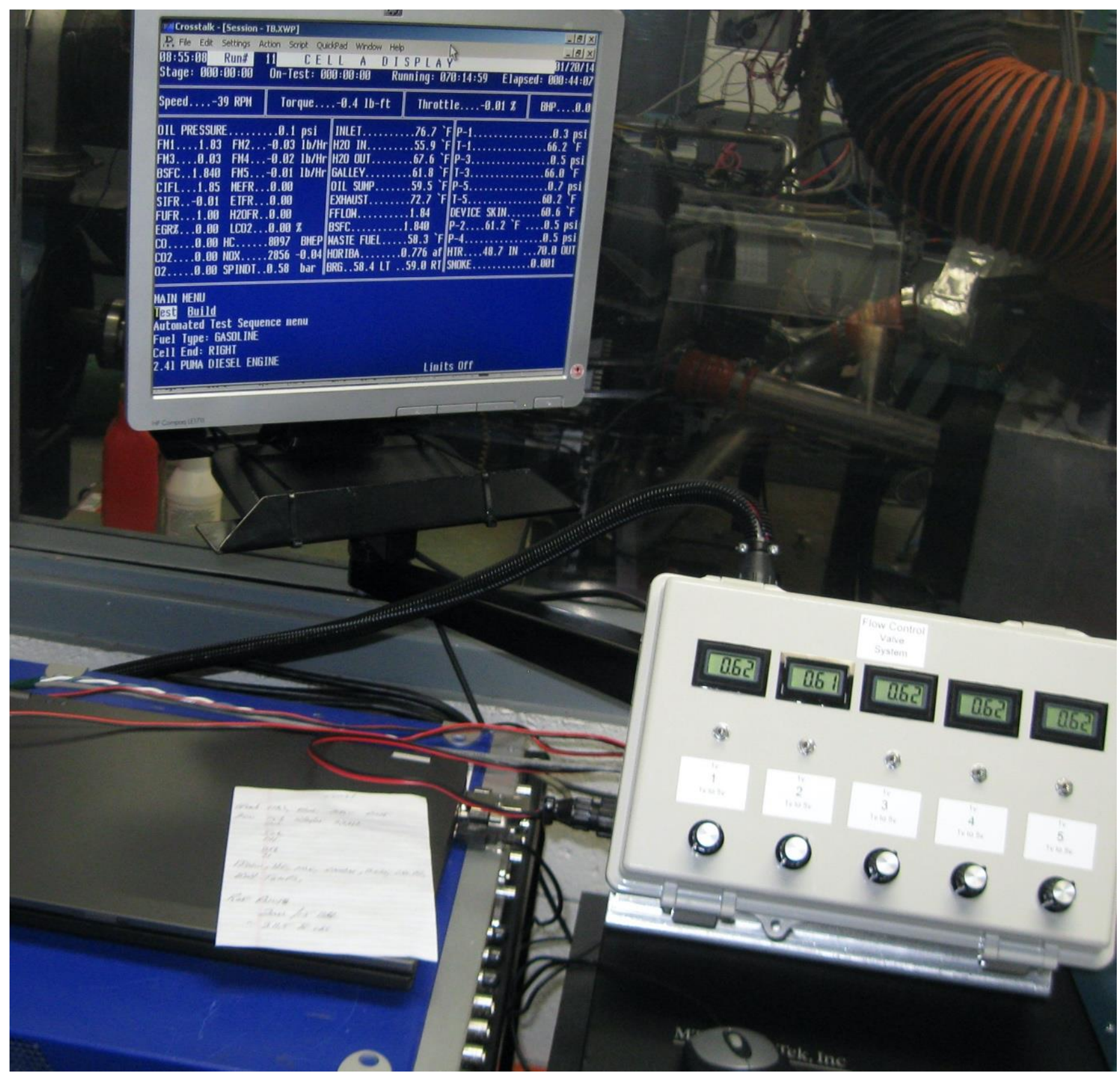

Figure 3, - the figure shows an example of the location of the system of analytical and control equipment of the test bench, intended for testing and analytical evaluation of a diesel engine. Bench systems include elements of artificial intelligence and artificial neural networks. It is very important in the application materials to show and disclose the novelty of the application of this system to achieve an ideal end result or its equivalent in relation to the claimed material 


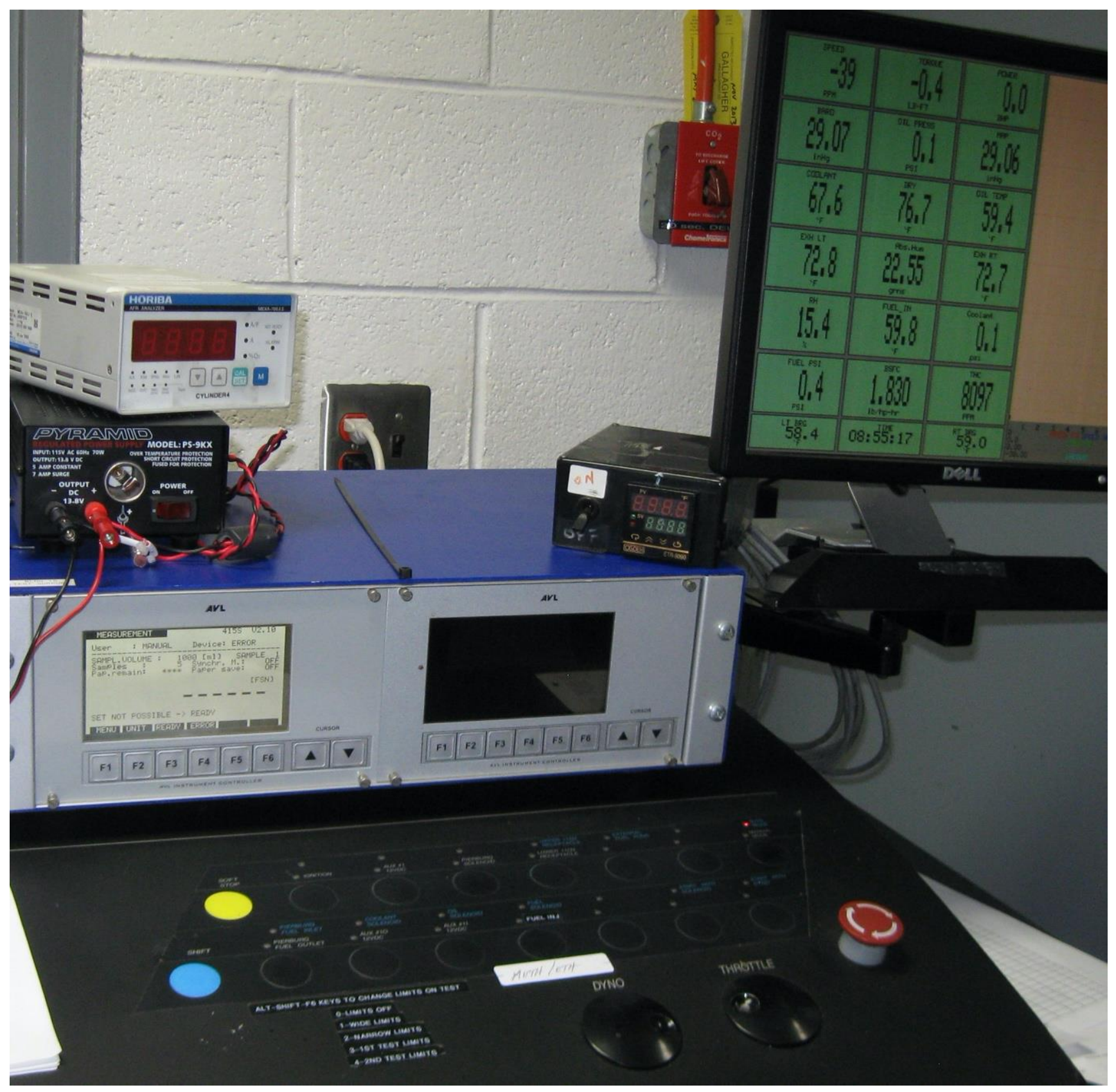

Figure 4, - the figure shows an example of a fragment of the location of the system of analytical and control equipment of the test bench, intended for testing and analytical evaluation of a diesel engine. Bench systems include elements of artificial intelligence and artificial neural networks. It is very important to show and disclose the novelty of using this system to achieve an ideal end result or its equivalent in relation to the claimed material, as well as to show the interaction of the control and analytical components of the system with elements of artificial intelligence 

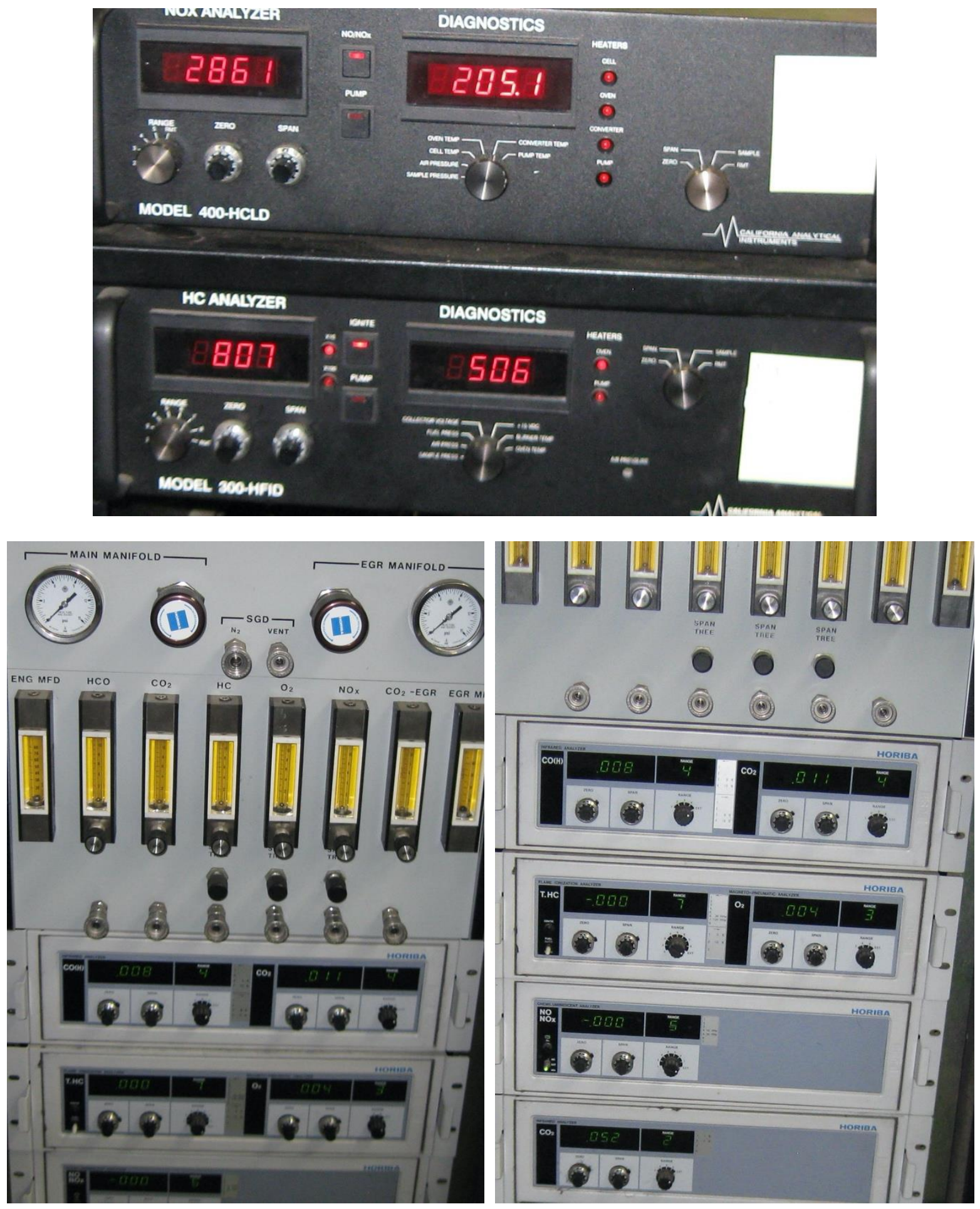

Figures 5, 6, 7, - the figures show examples of fragments of the location of the system of analytical and control equipment of the test bench, intended for testing and analytical evaluation of a diesel engine. It is very important to show and reveal the novelty of the application of this system, including the control and analytical components of the system with elements of artificial intelligence 


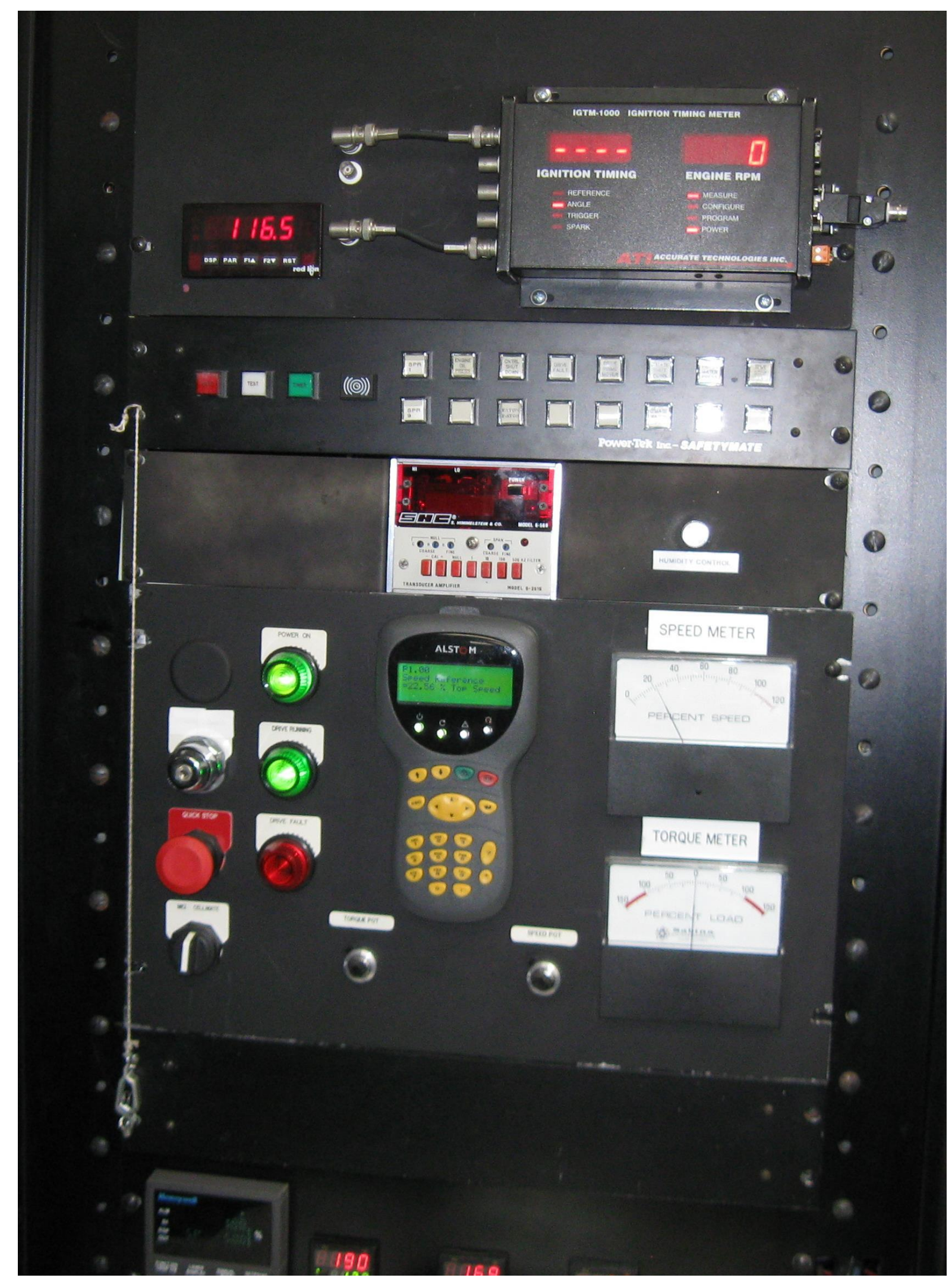

Figure 8 
Figures 8, 9, 10 and 11 show examples of fragments of the location of the system of analytical and control equipment of the test bench, intended for testing and analytical evaluation of a diesel engine. It is very important to show and reveal the novelty of the application of this system, including the control and analytical components of the system with elements of artificial intelligence.

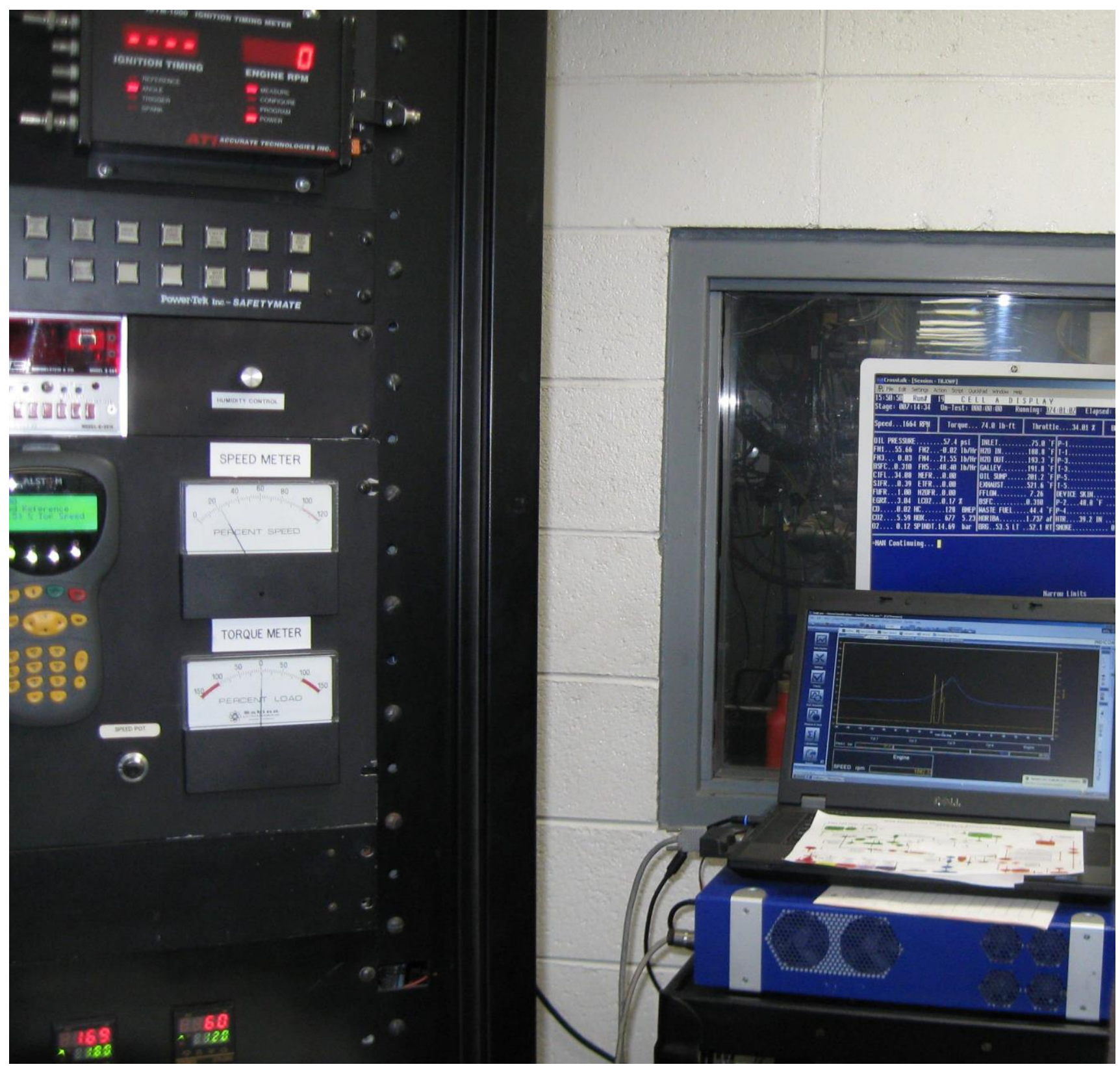

Figure 9 


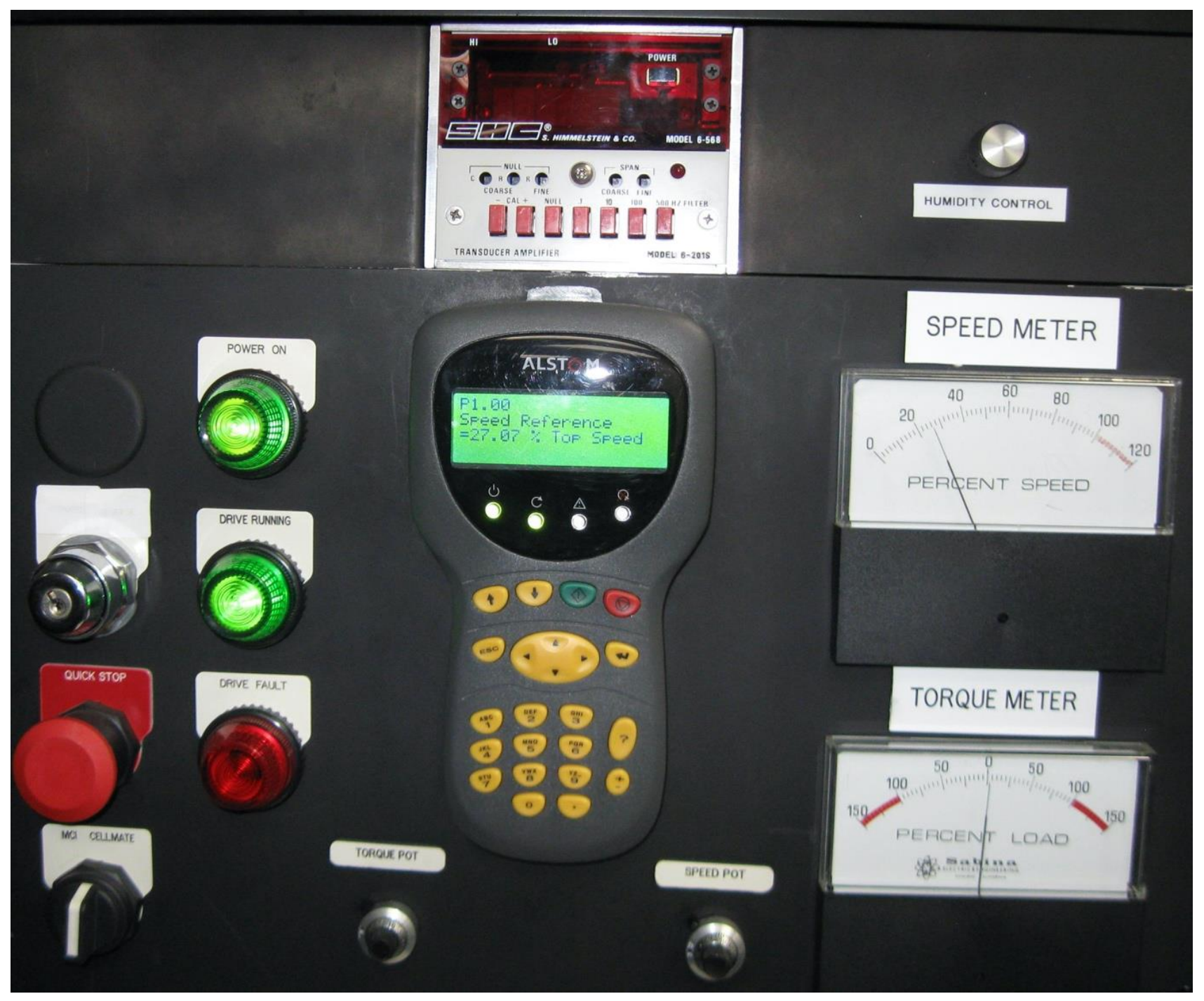

Figure 10, - the figure shows, as an example, the components of a control and analytical system that allow, in the process of comparative evolution of measurement results and comprehensive monitoring of the whole complex of technological transitions, to use the capabilities and characteristics of elements of artificial intelligence and artificial neural networks

This is especially important in the constant coordination of the main operating parameters of the test bench and the response from these parameters to the test components of the engine installed on the dynamometer table of the bench.

In the section in which the test results are given, it is necessary to connect the novelty elements of the modified engine components with the determination of their real indicators and the determination of the effect of this novelty on the nature of the indicators. 


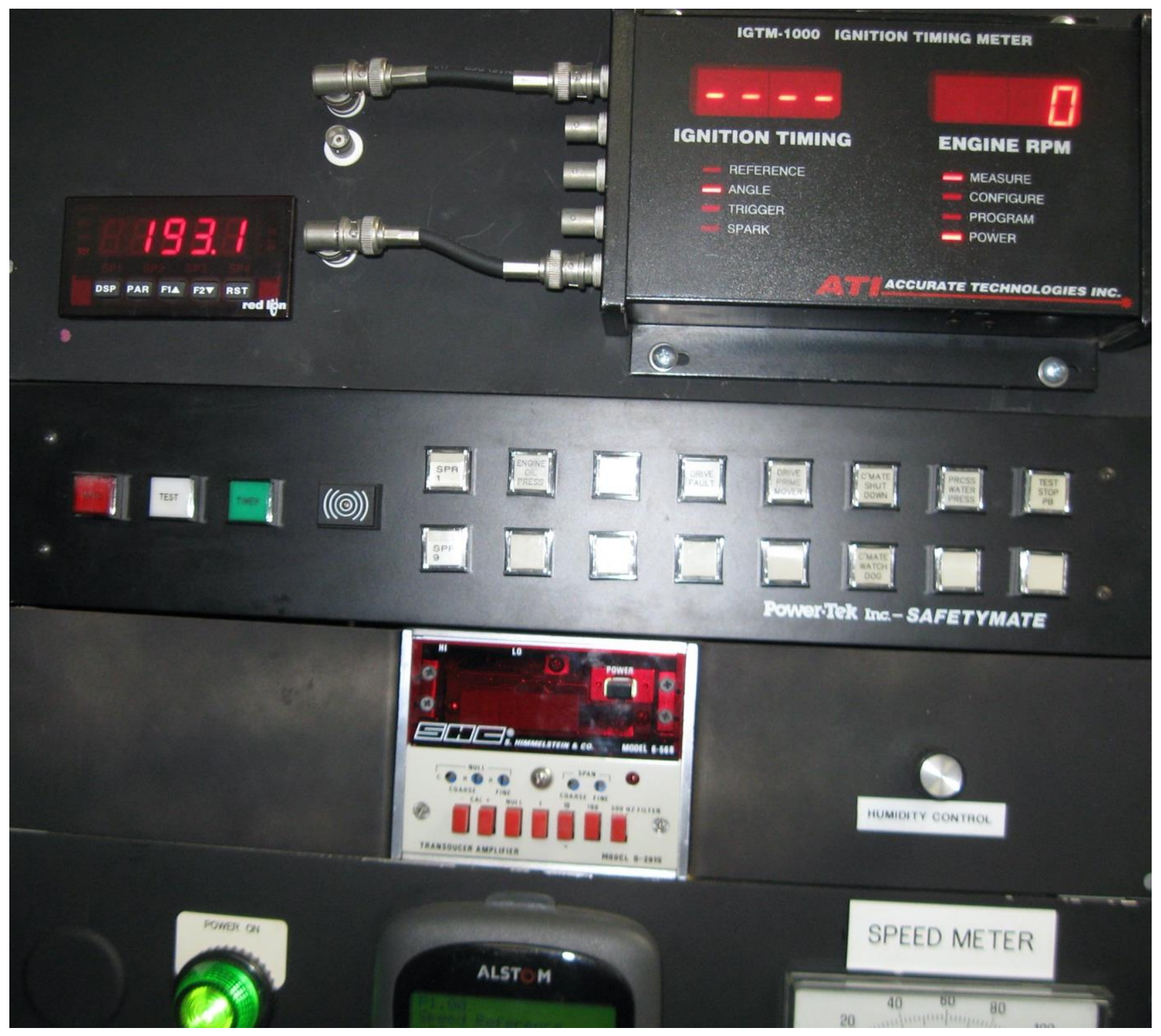

Figure 11, - the figure also shows, as an example, the components of the control and analytical system that allow the capabilities and characteristics of artificial intelligence elements and artificial neural networks to be used in the process of comparative evolution of measurement results and integrated monitoring of the entire complex of technological transitions

In the section in which the test results are given, it is necessary to connect the novelty elements of the modified engine components with the determination of their real indicators and the determination of the effect of this novelty on the nature of the indicators. 


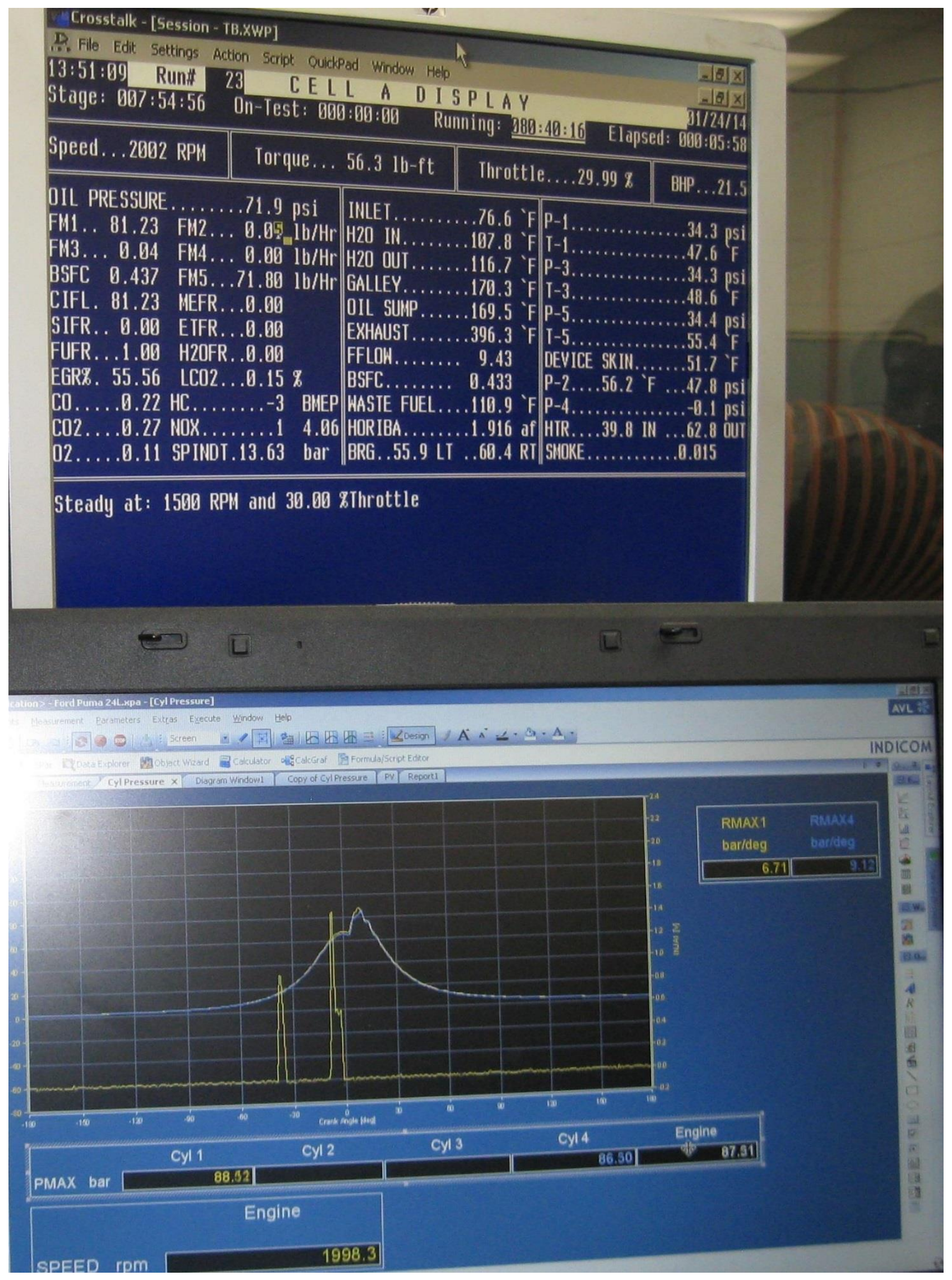


Figure 12

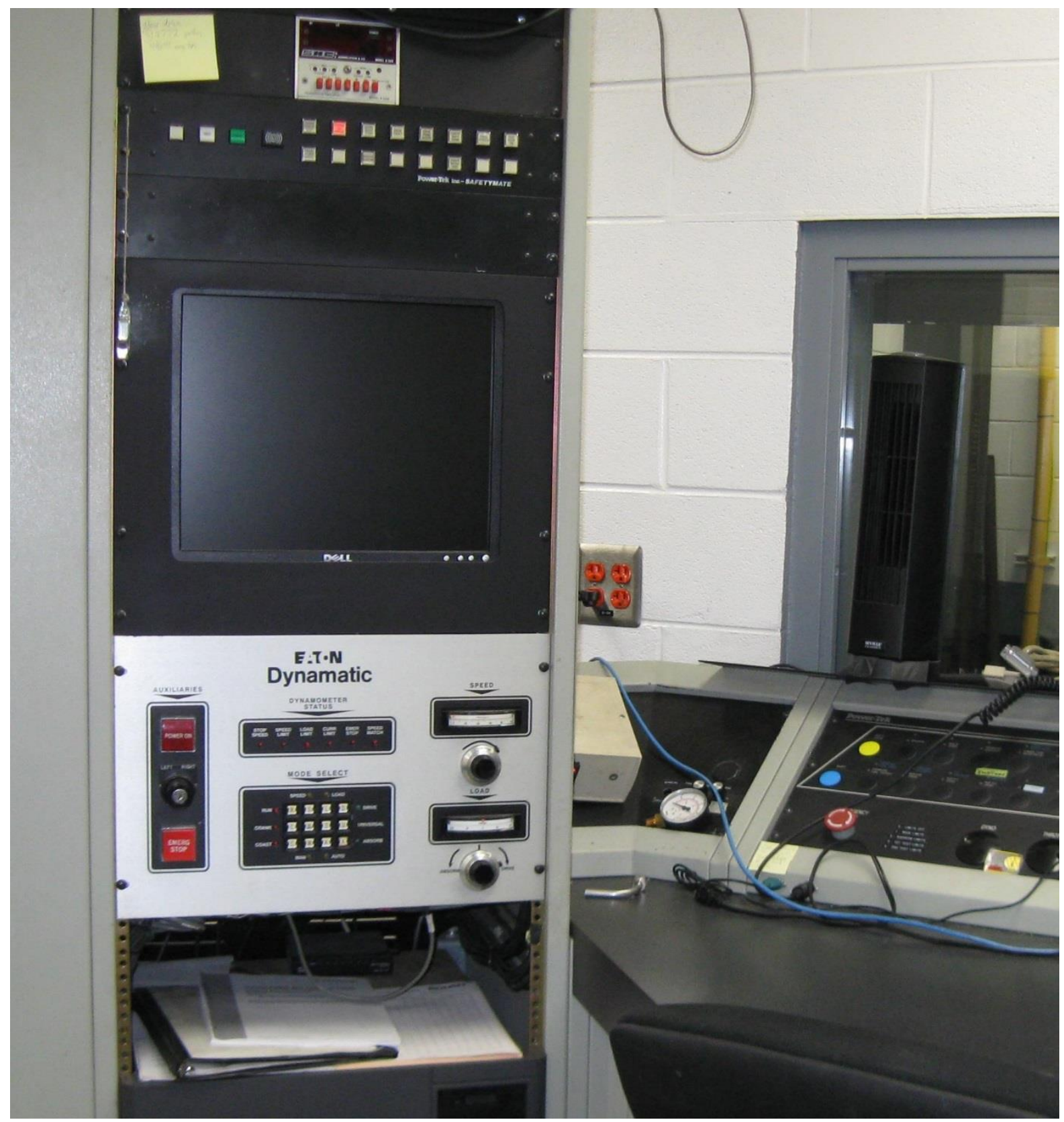

Figure 13

Figures 12, 13, 14, and 15 show the control and measurement processes that determine the nature of the interaction between various devices and measurement systems and their coordination with elements of artificial intelligence and artificial neural networks in terms of optimizing the main parameters of the technical characteristics of the engine, including environmental indicators associated with with the formation of fuel mixtures, including methanol or biological fuels. 


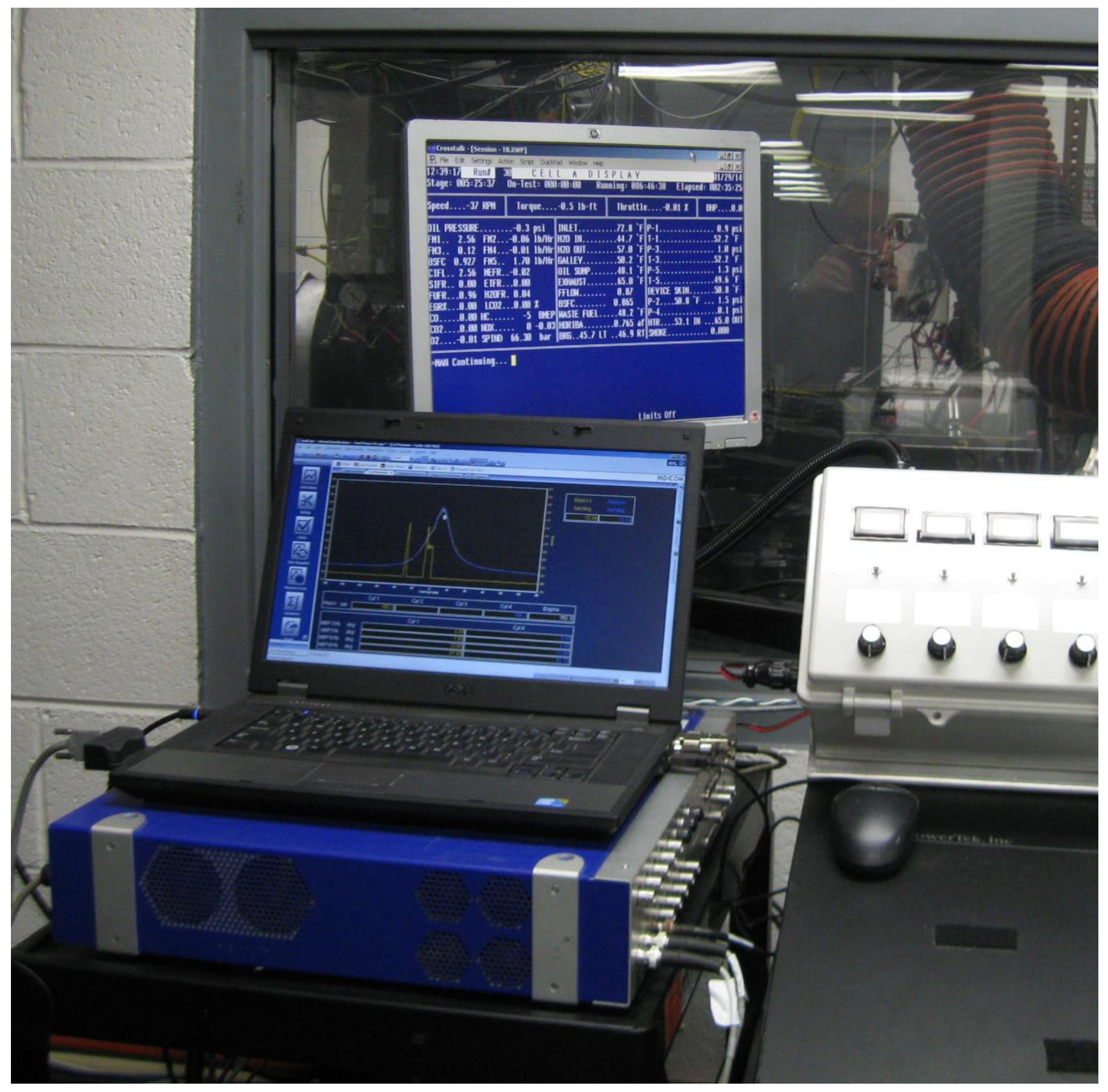

Figure 14

For applications for the proposed invention, it is important to show the influence of elements and signs of significant novelty of the proposed technical solution in their active combination with elements of artificial intelligence and artificial neural networks in combination with an ideal end result aimed at obtaining an unusual test result that does not take place in known technical solutions and characterizing the proposed technical solution. 


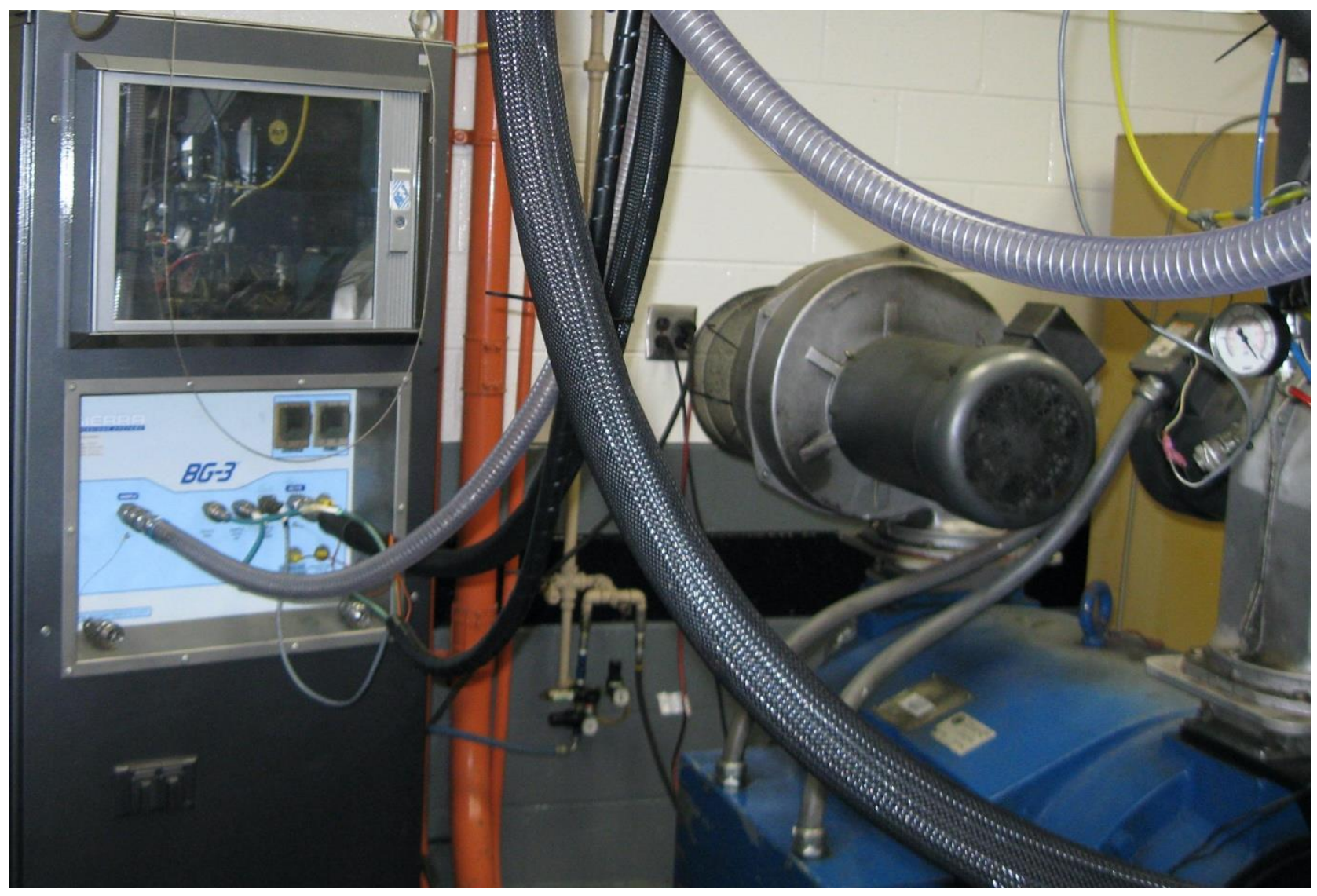

Figure 15

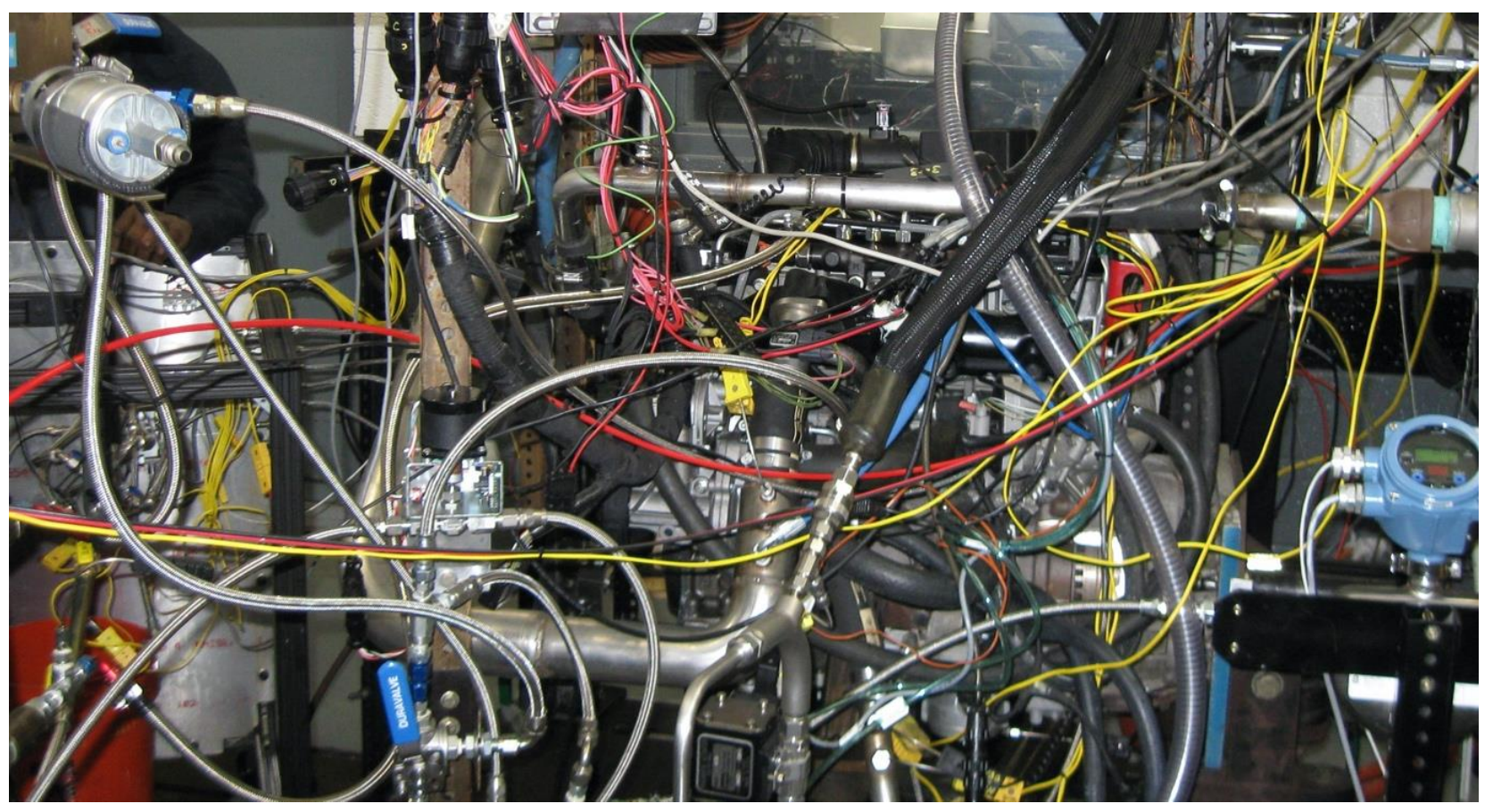




\section{Figure 16}

Figure 16 shows the engine on the stand, which controls more than 1200 parameters, the processing of the measurement results of which is carried out in real time using elements of artificial intelligence and artificial neural networks.

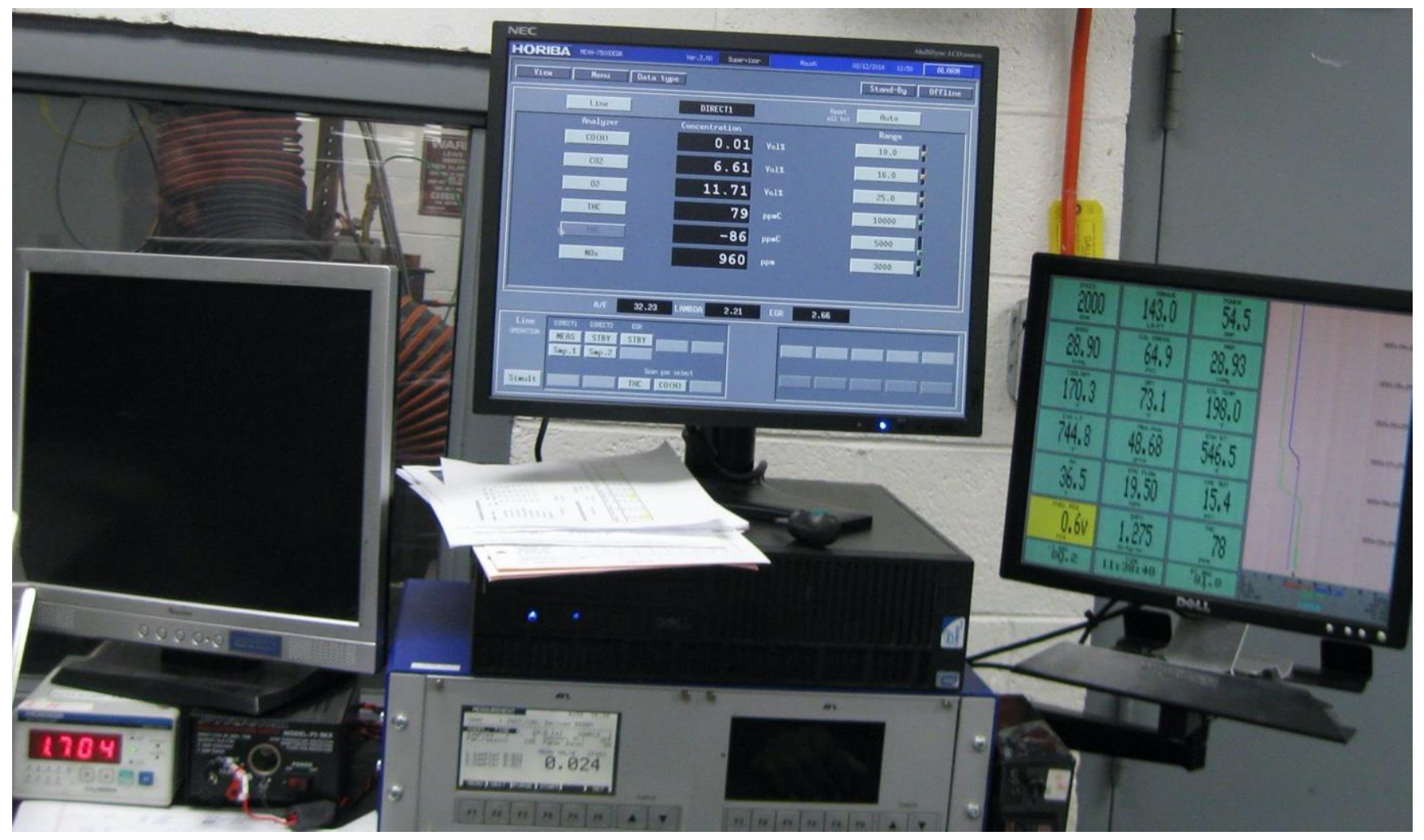

Figure 17, - the figure shows, as an example, the components of a control and analytical system that allow, in the process of comparative evolution of measurement results and comprehensive monitoring of the whole complex of technological transitions, to use the capabilities and characteristics of elements of artificial intelligence and artificial neural networks

This is especially important in the constant coordination of the main operating parameters of the test bench and the response from these parameters to the test components of the engine installed on the dynamometer table of the bench.

In the section in which the test results are given, it is necessary to connect the novelty elements of the modified engine components with the determination of their real indicators and the determination of the effect of this novelty on the nature of the indicators. 


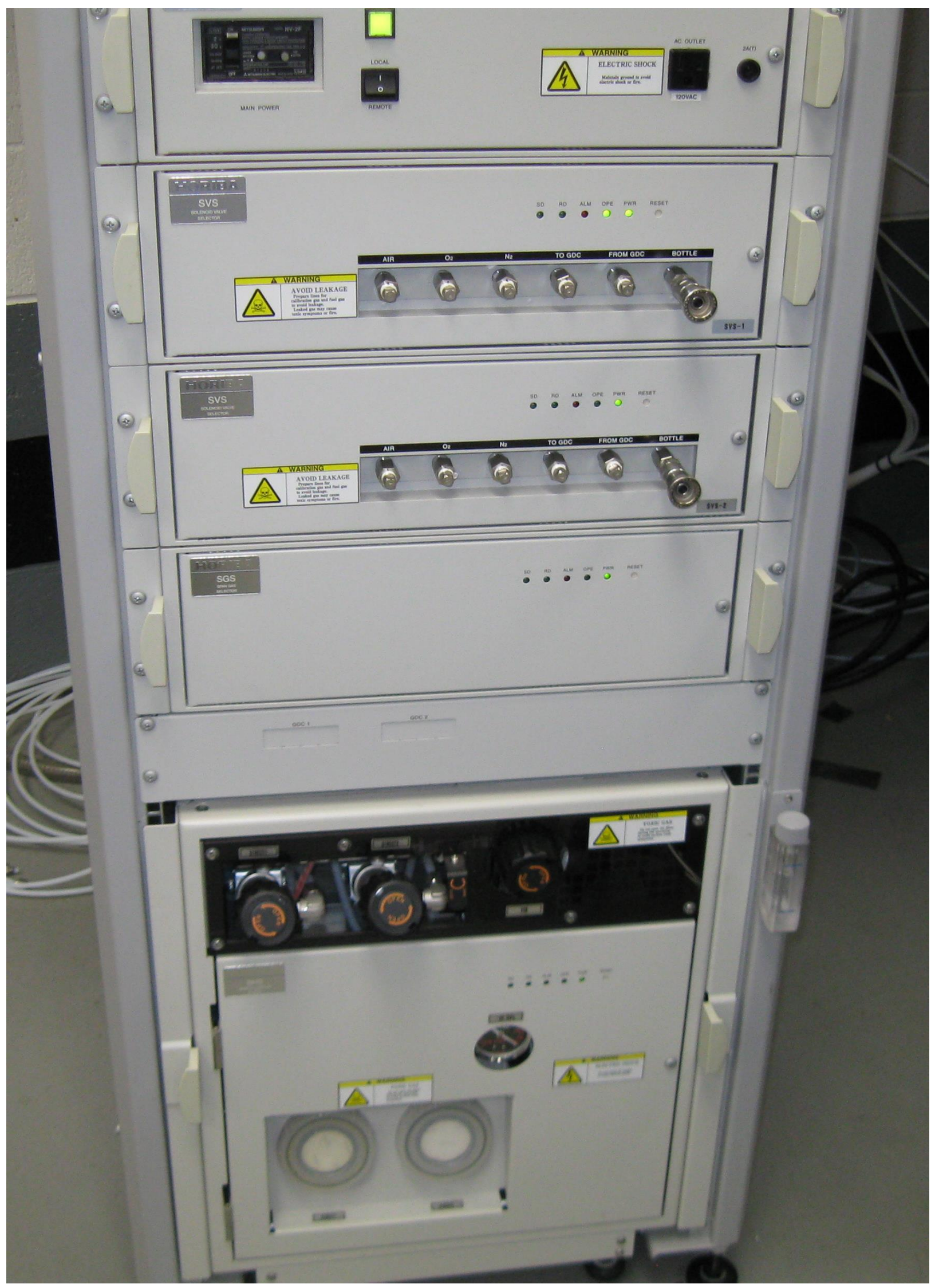




\section{Figure 18}

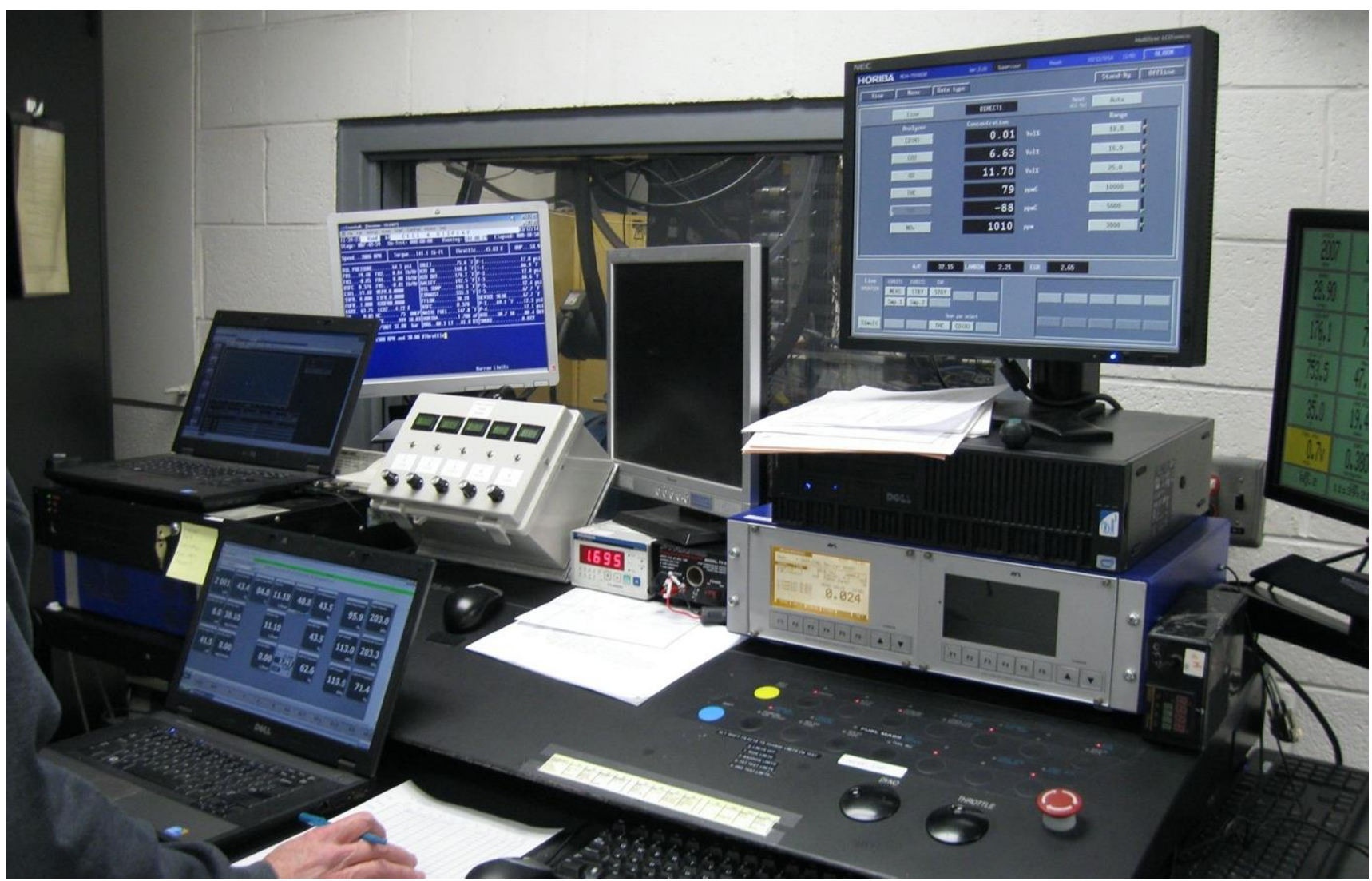

Figure 19

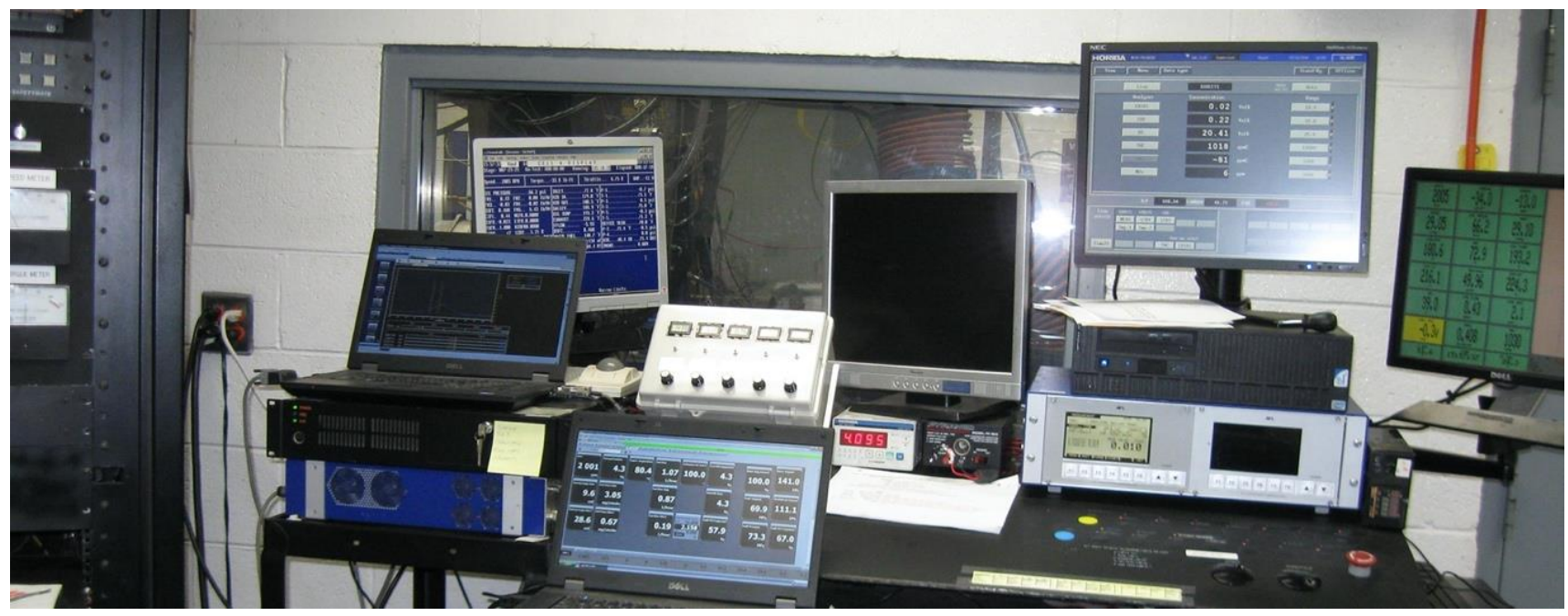

Figure 20 


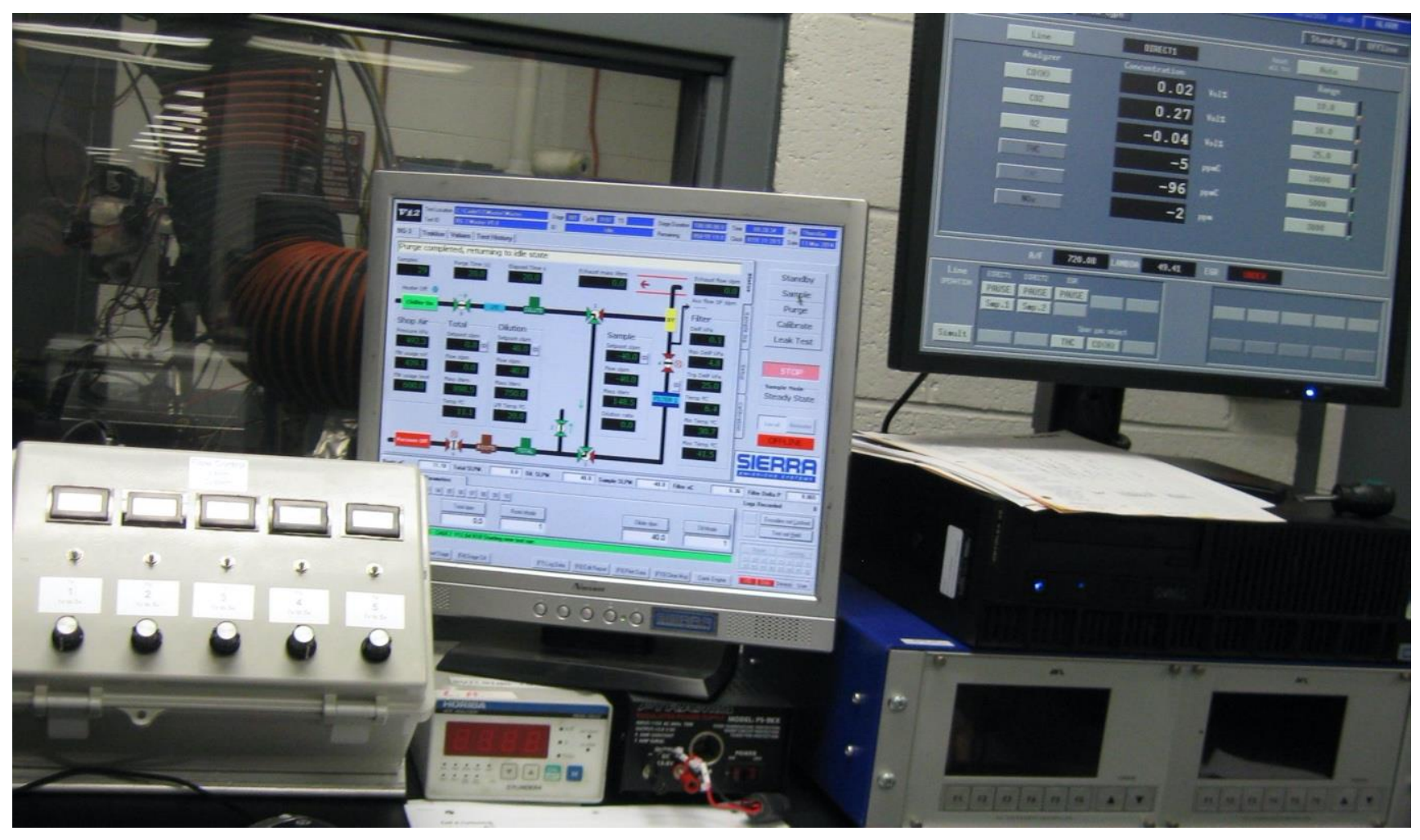

Figure 21

Figures 18, 19, 20, 21, 22, 23, and 24, - also show, as an example, the components of a controlanalytical system that are functionally connected with each other and simultaneously with elements of artificial intelligence and artificial neural networks, allowing in the process of comparative evolution of measurement results and integrated monitoring of the whole complex of technological transitions also comprehensively apply the capabilities and characteristics of elements of artificial intelligence and artificial neural networks.

This is especially important in the constant coordination of the main operating parameters of the test bench and the response from these parameters to the test components of the engine installed on the dynamometer table of the bench.

In the section in which the test results are given, it is necessary to connect the novelty elements of the modified engine components with the determination of their real indicators and the determination of the effect of this novelty on the nature of the indicators. 


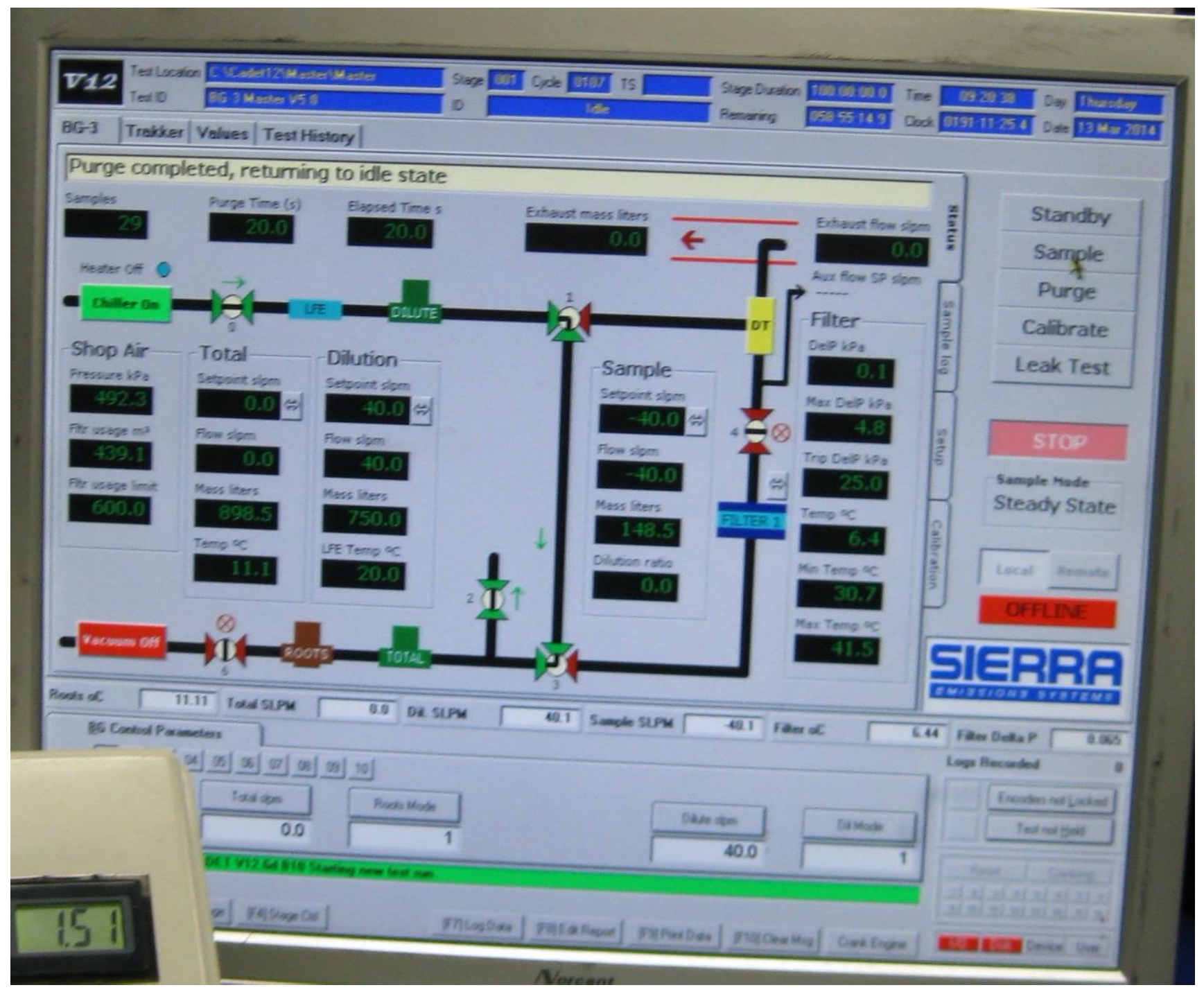

Figure 22

For the given example, indicators and combinations of measurement results of various, interconnected engine operation parameters, which vary depending on the factor that affects the result to the maximum extent, are typical, moreover, this may be the composition of the fuel mixture, including only liquid components, or liquid and gaseous components or only gaseous components.

A constant analysis of the effectiveness of all elements of novelty and their influence on the degree or level of achievement of an ideal end result to the maximum extent depends on the inclusion or not inclusion in the control and analytical complex of elements of artificial intelligence and artificial neural networks. 


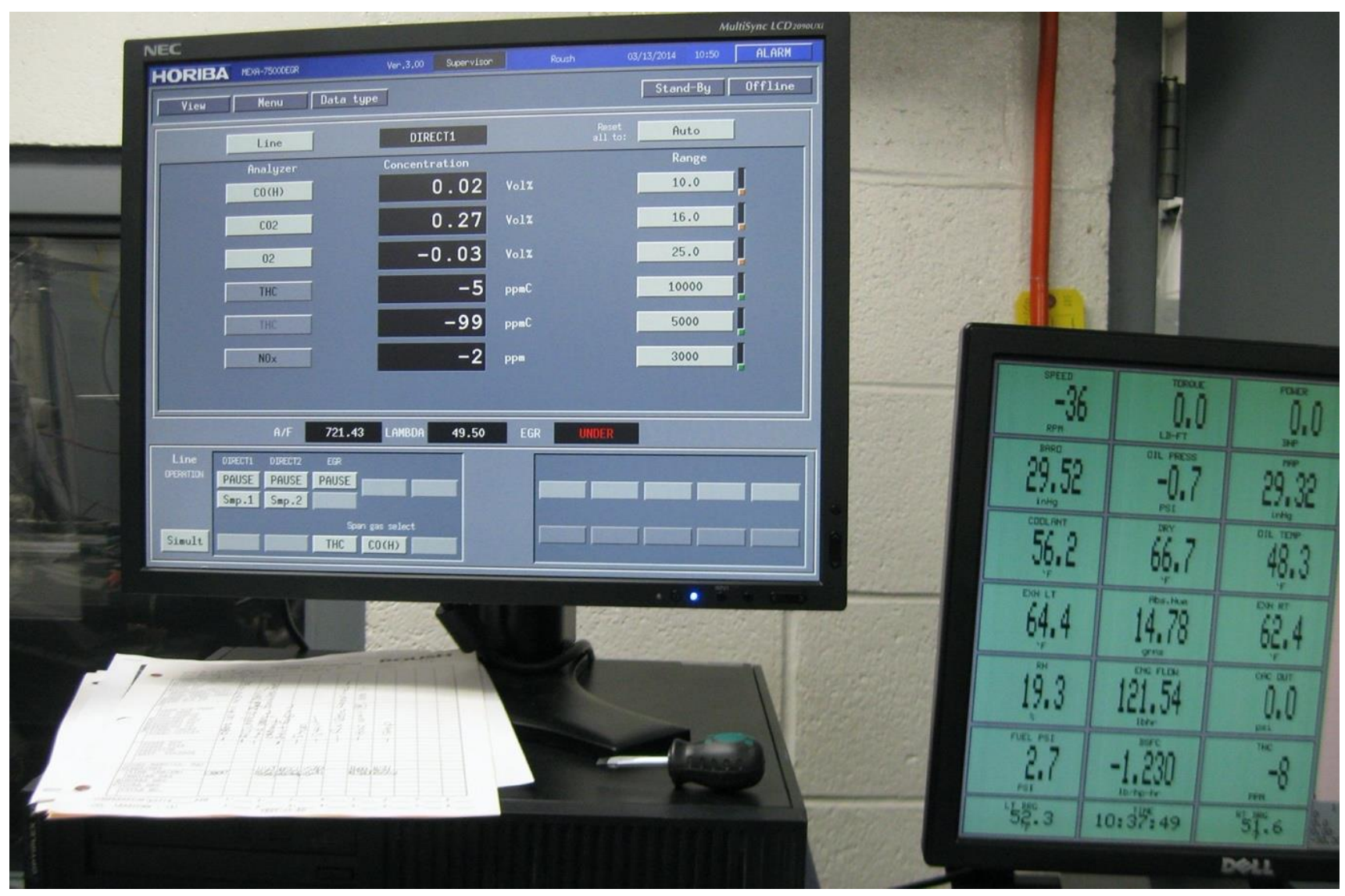

Figure 23

The task of inclusion and a detailed analysis of the impact of this inclusion is the material that should be included in the description of the application for the invention, in the section of evidence of the effectiveness of the elements of significant novelty and the results of their comprehensive tests using the above equipment.

In the text of the application related to the description of the test process and characterization of the test results, it is necessary to give a detailed detailed explanation of the principles of constructing the test process in combination with the analytical processing of the test results, including the connection with the requirements of the standards in force during the test period.

The description should include a characterization of the general distinguishing features of the alleged invention and their causal relationship with the results.

It is also necessary to indicate how new and non-obvious the results are. 


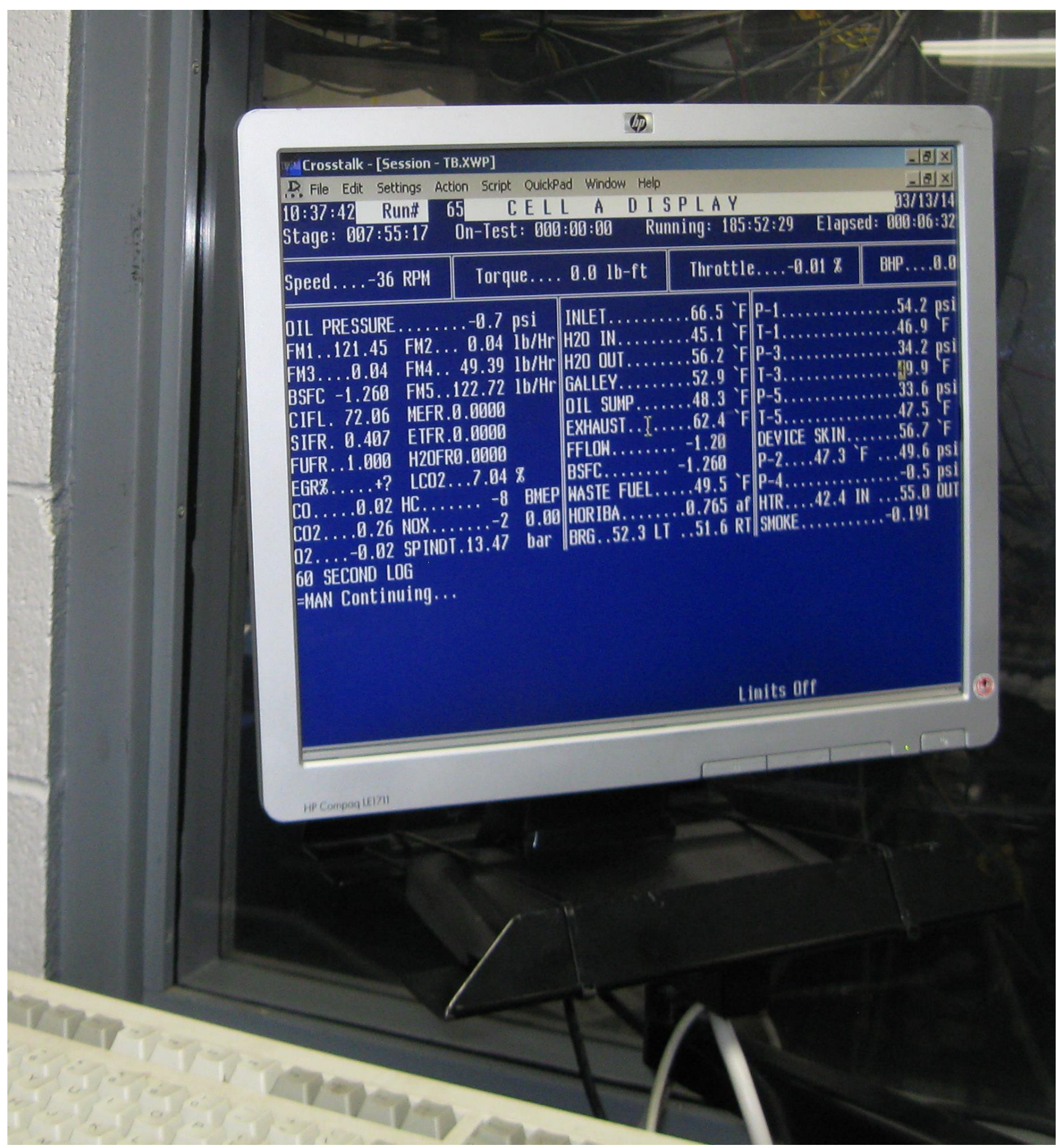

Figure 24

It is important to show the connection of the invention with smart technologies and the possibilities of combining software products of a new solution with the structural elements of an apparatus or system, forming a complex of new technical solutions that form the basis of the invention. 
[0085] Test results showed that in the..., - in this section, the test results are identified and compared with similar test results of known devices or apparatuses.

[0086] This section proves that the test results obtained correspond to the expected and declared indicators of the ideal final result.

\section{ANALYSIS OF THE TEST RESULTS}

[0087] Qualification test result analysis showed that the embodiments of the invention achieve the objective of..., - this section provides a structural analysis and characterization of the test results of prototypes of the invented engineering solution.

Next, you need to analyze the General distinguishing features of the invention, which distinguish it from known patent materials.

As an example, we consider an invention - an internal combustion engine, classified as a smart transport system for all structural elements and operating principles:

$\circ$ an internal combustion engine, into the fuel mixture supply and preparation system of which, a device for dynamically mixing liquid fuel with other components of the fuel mixture is integrated;

o the operating cycle of the device for dynamically mixing liquid fuel with other components of the fuel mixture occurs in the fuel pipeline with the main parameters of the fuel pipeline that occur in the normal duty cycle of any internal combustion engine;

$\circ$ in the operation of an engine with an integrated device for dynamically mixing the liquid component of the fuel mixture with other components of the fuel mixture, only materials and substances are used that are commonly used in the operation of serial internal combustion engines;

- a device for dynamic mixing of components has two complex input devices sequentially arranged along the course of the fuel, ending with vortex generators forming a vortex tube within the fuel pipeline;

$\circ$ the device for dynamically mixing the components of the fuel mixture has a mixing system, which when mixing equalizes the level of turbulence in each cross section of the fuel pipeline in the center of the cross section of the pipeline and on its periphery;

$\circ$ the fuel mixture at the outlet of the device for dynamic mixing of the components of the fuel mixture, depending on the mixing ratio between the components, and depending on the nature and type of components, may have a different structure and composition:

○ emulsions

$\circ$ foamed emulsion

o foamed fuel mixture 
○ fuel foam

○ aerosols

○ carbonated fuel.

In none of the known technical solutions does the fuel mixture have such a composition and structure before being injected into a cylinder or combustion chamber.

If gas acts as a component of the fuel mixture, due to the fact that in the device for dynamically mixing liquid fuel with other components of the fuel mixture, the contact surface area in the boundary layer — liquid / gas — increases by at least an order of magnitude compared with opposed technical solutions (at least 10 times) the concentration of dissolved gas in a liquid increases in proportion to the increase in contact area:

$\circ$ for mixing, only materials and components, both liquid and gaseous, which are traditionally used for the preparation of fuel mixtures, are used;

O for the preparation of fuel mixtures in the proposed technical solution, only known technological methods and actions are applied ..., only hydrodynamic and aerodynamic mixing and technological methods and actions are not applied, as in well-known patent applications:

○ "Fuel converter" - the declared effect is achieved due to heating, evaporation and catalysis of the fuel mixture, and not due to only hydrodynamic and aerodynamic mixing, as is the case in the proposed technical solution. In the known material there are no signs inherent in the technical solution underlying the present invention and, based on this, the known technical solution is not the technical equivalent of the claimed technical solution according to any of the signs and criteria.

○ "Heated catalyzed fuel injector for injection ignition engines", the declared effect is achieved due to additional compression and heating of the fuel mixture and additional catalytic effect on the fuel mixture, and not due to only hydrodynamic and aerodynamic mixing of the components of the fuel mixture, as in the proposed technical solution. In the known material there are no signs inherent in the technical solution underlying the present invention and, based on this, the known technical solution is not the technical equivalent of the claimed technical solution according to any of the signs and criteria.

○ "Single nozzle direct injection system for rapidly variable gasoline / anti-knock agents mixtures", the declared effect is completely different from the effect of the proposed technical solution. The goal of the well-known technical solution ..., is mixing an antiknock agent with the fuel mixture, which reduces the level of gasoline detonation. In this case, mixing is carried out according to a technology that is absolutely not equal and not equivalent in any way to the claimed technical solution; In the known material there are no signs inherent in the technical solution underlying the present invention, and, based on this, the 
opposed technical solution is not the technical equivalent of the claimed technical solution according to any of the signs and criteria.

○ "Internal combustion engine / water sours system", the declared effect is the cooling of exhaust gases in order to condense water from them, it is proposed to carry out by compressing the exhaust gases and forming a vortex tube from them. Based on the materials set forth in the well-known technical solution, we can conclude that it is impossible in this technical solution to reduce the temperature of the exhaust gases to such a level that condensation of water is possible; Known technical solution is absolutely not functional. In the known material there are no signs inherent in the technical solution underlying the present invention and, based on this, the known technical solution is not the technical equivalent of the claimed technical solution according to any of the signs and criteria.

Based on the test results of the prototype of the proposed technical solution integrated into the fuel system of a serial diesel engine, the result is new for the actual practice of operating diesel engines and amounts to $-97 \%$ reduction in the level of soot resulting from the combustion of the fuel mixture in the engine cylinders.

Such a result is completely new for both diesel and gasoline engines, and none of the known technical solutions in any of its signs solves the equivalent technical problem at such a level, which means that the known technical solutions in relation to the proposed technical a solution with proven full performance and documented efficiency, for all four well-known technical solutions with undetermined performance and with unproven efficiency is I technically incompetent. In addition, the prevention of soot formation in engine cylinders due to only hydrodynamic and aerodynamic mixing of standard components of the fuel mixture, which is not known from previous publications and inventions and is also absent in known technical solutions, is in relation to each of the known technical solutions, separately and all opposed technical solutions in the aggregate, completely new.

\section{EXAMPLES OF APPLICATIONS}

[0088] In some embodiments..., - this section provides examples of the various applications of the invention or the various embodiments of the invention, if any.

If a variety of embodiments and applications are not included in the main distinctive features of the claims, it is very important to detail them more clearly and more precisely.

In the future, when identifying and verifying the fact of actual use of the invention, these application samples help to more convincingly prove the facts of use of the invention. 
[0089] In some embodiments..., - this section provides examples of the various applications of the invention or the various embodiments of the invention, if any.

If a variety of embodiments and applications are not included in the main distinctive features of the claims, it is very important to detail them more clearly and more precisely.

In the future, when identifying and verifying the fact of actual use of the invention, these application samples help to more convincingly prove the facts of use of the invention.

[0090] In some embodiments..., - this section indicates thanks to what peculiar features and combinations of features in each of the considered embodiments, the achievement of the ideal final result is provided, which is expected from this particular embodiment.

[0091] In some embodiments..., - this section indicates thanks to what peculiar features and combinations of features in each of the considered embodiments, the achievement of the ideal final result is provided, which is expected from this particular embodiment.

[0092] In some embodiments..., - this section indicates thanks to what peculiar features and combinations of features in each of the considered embodiments, the achievement of the ideal final result is provided, which is expected from this particular embodiment.

[0093] In some embodiments..., - this section indicates thanks to what peculiar features and combinations of features in each of the considered embodiments, the achievement of the ideal final result is provided, which is expected from this particular embodiment.

[0094] In some embodiments..., - this section indicates thanks to what peculiar features and combinations of features in each of the considered embodiments, the achievement of the ideal final result is provided, which is expected from this particular embodiment.

[0095] In some embodiments..., - this section indicates thanks to what peculiar features and combinations of features in each of the considered embodiments, the achievement of the ideal final result is provided, which is expected from this particular embodiment.

[0096] In some embodiments..., - this section indicates thanks to what peculiar features and combinations of features in each of the considered embodiments, the achievement of the ideal final result is provided, which is expected from this particular embodiment.

[0097] In some embodiments..., - this section indicates thanks to what peculiar features and combinations of features in each of the considered embodiments, the achievement of the ideal final result is provided, which is expected from this particular embodiment.

[0098] In some embodiments..., - this section indicates thanks to what peculiar features and combinations of features in each of the considered embodiments, the achievement of the ideal final result is provided, which is expected from this particular embodiment. 
[0099] An operating principle of the devices is..., - this section analyzes and compares the options for operating an apparatus or device, analyzes the options for management and control processes, and application of the principles of forming feedback elements between actuators and control systems or processors.

[0100] This section compares the capabilities and features of the various listed options and examples of application of the invention.

[0101] In some embodiments..., - this section indicates thanks to what peculiar features and combinations of features in each of the considered embodiments, the achievement of the ideal final result is provided, which is expected from this particular embodiment.

[0102] In some embodiments..., - this section indicates thanks to what peculiar features and combinations of features in each of the considered embodiments, the achievement of the ideal final result is provided, which is expected from this particular embodiment.

[0103] It will be appreciated that the..., - this section indicates thanks to what peculiar features and combinations of features in each of the considered embodiments, the achievement of the ideal final result is provided, which is expected from this particular embodiment.

[0104] Various embodiments of the invention allow one or more of the following advantages..., - this section identifies and analyzes any advantages arising from the embodiment of the invention.

[0105] It will be appreciated by those skilled in the art that various omissions, additions and modifications may be made to the methods and structures described above without departing from the scope of the invention. All such modifications and changes are intended to fall within the scope of the invention, as defined by the appended claims.

As an example of an analytical assessment, we give a comparative characteristic of an innovative product, which in general can be represented as, for example, a fuel mixture, is as follows:

○ the product is formed within a short period of time - this time can be equal to at least a few milliseconds, for which the product receives all the necessary characteristics and properties and immediately becomes suitable for further use;

○ the product retains its properties for a long time if the pressure under which it is formed does not change or if it increases;

- the product is able to maintain its properties and characteristics even with a sharp increase in pressure (during testing, the product pressure was up to 2000 bar and no changes in the structure and properties of the product were found);

○ the product has a flexible hydrodynamic structure;

$\circ$ the product has a flexible chemical structure; 
- the product can consist entirely of homogeneous liquids and gases in their properties, while all liquid and gaseous components can be mixed in various proportions and combinations;

○ the product can consist of liquids and gases that are heterogeneous in properties, while all liquid and gaseous components can be mixed in various proportions and combinations;

$\circ$ after formation, various chemicals that are not part of the starting product may be added to the product;

$\circ$ various chemicals that are not part of the starting material may be added to the product during formation.

An invented product can have many varieties. The varieties of the invented product include:

a homogeneous mixture consisting of at least two homogeneous liquids;

- a homogeneous mixture consisting of at least two dissimilar liquids;

○ a mixture consisting of at least two homogeneous liquids and at least one gas;

$\circ$ a mixture consisting of at least two dissimilar liquids and at least one gas;

- a mixture consisting of at least one solution of liquids and at least one additional liquid;

- a mixture consisting of at least two homogeneous solutions of liquids;

- a mixture consisting of at least two heterogeneous solutions of liquids;

- a mixture consisting of at least two homogeneous solutions of liquids and at least one gas;

$\circ$ a mixture consisting of at least two heterogeneous solutions of liquids and at least one gas.

Chemical reagents can be introduced into the mixture both during formation and after formation. These types of mixtures can also have many varieties:

- a mixture consisting of at least two homogeneous liquids with the addition, after formation, of additional chemicals;

- a mixture consisting of at least two dissimilar liquids with the addition of additional chemicals after formation;

- a mixture consisting of at least one liquid solution and at least one liquid with the addition of additional chemicals after formation;

- a mixture consisting of at least two homogeneous solutions of liquids with the addition of additional chemical reagents after formation;

- a mixture consisting of at least two heterogeneous solutions of liquids with the addition of additional chemical reagents after formation.

Further it is necessary to reveal the essence of technology, that underlies the formation of a new product - by way of example we give a mixture, which are the production technology is as follows: 
$\circ$ at the moment of formation of the mixture, the level of turbulence at all points of the cross-section of the flows of the basic components of the mixture is equalized, due to which the necessary level of uniformity of state over the entire volume and the required level of particle size of the components of the mixture are achieved;

$\circ$ the kinetic energy of the particles of all components of the mixture is conserved, and this energy is released if the pressure under which the mixture is reduced or even becomes atmospheric;

$\circ$ the release of the kinetic energy of the components of the mixture helps (as, for example, during the combustion process to obtain the maximum atomization of the fuel liquid component of the mixture);

O the number of components in the mixture is practically unlimited, since the apparatus for forming the mixture can be adapted to the required number of components without changing the fundamental principles of the technology;

$\circ$ if a gas or a mixture of gases is used as one or several components, the fundamental principles of the technology make it possible to obtain an aerosol in the form of a mixture or allow mixing the mixture with compressed gas or air;

○ the technology allows sequential introduction of additional components into the mixture, and the number of such components and their chemical composition are not limited;

O since all the parameters of the mixture depend on the pressure in the flow of the components of the mixture, the regulation of the properties and parameters of this mixture can be carried out by adjusting the pressure.

\section{WHAT IS CLAIMED IS...}

1. The first independent claim is the most important for the application:

The first claim should be extremely brief and at the same time consist of three parts:

$\circ$ The first part gives the commercial name of engineering solution, which should cover the largest possible area of commercial use, and at the same time give an idea of the scope of the claims of the claimed engineering solution, excluding advertising manner of speech and paradoxical statements;

- The second part of the first claim describes the distinctive features, their sets and interrelations, which are known and used as basic for the realization of distinctive features;

- The third part of the first claim describes the distinctive features, their sets and static and dynamic relations of the distinctive features with the basic known features, which together ensure the achievement of an ideal final result. 
Subsequent dependent claims should describe in more detail the distinctive features in the most general form. All subsequent claims should disclose all possible applications of distinctive features to form an effect and achieve an ideal final result. If the ideal final result can be achieved in some options and subject to the availability of different options for combining distinctive features with basic features, all possible options are indicated in the following claims.

Each subsequent claim also consists of three parts, and the requirements for each of them corresponds to the requirements for the first claim.

2. The second independent claim of the invention.

3. The claim dependent on the first and third claims of the invention.

4. The claim dependent on the first and third claims of the invention.

5. The dependent on the first and third claims of the invention.

6. The dependent on the first and third claims of the invention.

7. The third independent claim of the invention.

If the subject of the invention, - a device or apparatus and the named, - is an apparatus, for example, it is preferable to have at least three independent claims, so that the first claim is, - apparatus for..., method of application and associated method of manufacture.

The third claim, - the method of the apparatus application.

8. The eighth claim - the apparatus for... and associated method of manufacture.

All of the above is just an example, each engineering solution has its own peculiar features, and, accordingly, the composition and structure of the construction of the claims may change.

\section{ABSTRACT OF THE DISCLOSURE}

Abstract, in principle, is not special and there are no recommendations for its composition.

\section{LIST OF REFERENCES, PATENT AND LICENSE MATERIALS}

\section{ANNEX 1}




\title{
ARTIFICIAL INTELLIGENCE DATA PROCESSING SYSTEM AND METHOD
}

\begin{abstract}
There are provided a system that is capable of performing tasks associated with IPR procurement. The system employs a computing architecture that provides characteristics of artificial intelligence (AI). The computing architecture employs a configuration of pseudoanalog variable-state machines that is implemented by disposing the pseudo-analog variablestate machines in a hierarchical arrangement, wherein pseudo-analog variable-state machines higher in the hierarchical arrangement mimic behavior of a human claustrum for performing higher cognitive functions when processing information associated with one or more service requests and for performing quality checking of the one or more work products. Moreover, the computing architecture is susceptible to being implemented by employing a novel configuration of data processing devices, for example arrays of RISC processors.
\end{abstract}

\section{ANNEX 2}

United States Patent Application

20190205606

Kind Code

Zhou; Shaohua Kevin ; et al.

July 4, 2019

\section{METHOD AND SYSTEM FOR ARTIFICIAL INTELLIGENCE BASED MEDICAL IMAGE SEGMENTATION}

\begin{abstract}
Methods and systems for artificial intelligence based medical image segmentation are disclosed. In a method for autonomous artificial intelligence based medical image segmentation, a medical image of a patient is received. A current segmentation context is automatically determined based on the medical image and at least one segmentation algorithm is automatically selected from a plurality of segmentation algorithms based on the current
\end{abstract}


segmentation context. A target anatomical structure is segmented in the medical image using the selected at least one segmentation algorithm.

\title{
ANNEX 3
}

United States Patent Application

20190206218

Kind Code

A1

KUSENS; BRUCE HOWARD; et al.

July 4, 2019

\section{UTILIZING ARTIFICIAL INTELLIGENCE TO DETECT OBJECTS OR PATIENT SAFETY EVENTS IN A PATIENT ROOM}

\begin{abstract}
Methods and systems are provided for detecting objects or patient safety events in a patient room. Artificial intelligence is utilized to enhance safety issue recognition capabilities by the methods and systems. Sensors collect a series of images and depth data in a room of a patient. Data (corresponding to images and depth data of an object or patent safety event) is received from the sensors and compared to stored data to identify the object or patient safety event. The images are communicated to a central video monitoring system and a user may be prompted to confirm if the object requires learning or a patient safety event occurred (or identify the object or patient safety event) or to provide additional parameters or actions. A patient safety learning system analyzes the data and incorporates the user response to enhance safety issue recognition capabilities of the system and reduce false alerts.
\end{abstract}

\section{ANNEX 4}

United States Patent Application

20190189236

Kind Code

A1

Poliakov; Pavel ; et al.

June 20, 2019

\section{ARTIFICIAL INTELLIGENCE BASED MONITORING OF SOLID STATE DRIVES AND DUAL IN-LINE MEMORY MODULES}




\begin{abstract}
In embodiments, a memory controller (MC) includes an output interface, and an execution engine (EE) to identify, based on field test results of a die coupled to the $\mathrm{MC}$, initial test results of the die using an artificial neural network (ANN) trained to identify the die from a set of NVM dies based on initial test results of the set of NVM dies obtained at a time of manufacture of the set of dies. The initial test results include a first useful life prediction and the field test results include a second useful life prediction, and the initial test results are regenerated by the ANN to protect their confidentiality. In embodiments, the MC is further to compare the second useful life prediction with the first useful life prediction, to determine a deviation between the two, and output, via the output interface, the deviation to a user.
\end{abstract}

\title{
ANNEX 5
}

United States Patent Application

20190205885

Kind Code

A1

Lim; Chern Jie ; et al.

July 4, 2019

\section{MACHINE LEARNING ENGINE FOR FRAUD DETECTION FOLLOWING LINK SELECTION}

\begin{abstract}
A machine learning engine for fraud detection following link selection may be trained using artificial intelligence techniques and used according to techniques discussed herein. A buyer account may be used to establish and generate a digital gift card having a particular value specified by the buyer. The digital gift card may then be conveyed to another account, such as an email address. The digital gift card may be provided with an online electronic process for redemption and use of the value, for example, by selecting a link and navigating to the process. When the claimer account attempts to utilize the value of the gift card by navigating to the process or otherwise engaging in the electronic process through a device, a risk and fraud analysis engine may execute to determine, based on real-time data of the claimer account, the buyer account, and/or device, whether the digital gift card was generated fraudulently or is being used fraudulently.
\end{abstract}

\section{ANNEX 6}




\title{
DEVICE OF CONTROLLING RELATED DEVICE USING ARTIFICIAL INTELLIGENCE BASED ON OPERATION SITUATION, SCHEDULE BOT AND SERVER CONTROLLING THEREOF
}

\begin{abstract}
This application is a technology with regard to a device that controls a related device based on an operation situation using artificial intelligence, a schedule bot and a server that controls the same, and the device that receives a control of the related device based on the operation situation by an exemplary embodiment of this application includes a function unit that performs a physical function of the device, a communication unit that receives a time schedule and function information from a related device or a schedule management device, and control unit that controls the function unit to maintain a ready state so that a function unit performs a first function at a point that is indicated in the time schedule by using the time schedule and the function information that the communication unit receives.
\end{abstract}

\section{ANNEX 7}

United States Patent Application

20190197357

Kind Code

A1

ANDERSON; GLEN J. ; et al.

June 27, 2019

\section{MISUSE INDEX FOR EXPLAINABLE ARTIFICIAL INTELLIGENCE IN COMPUTING ENVIRONMENTS}

\begin{abstract}
A mechanism is described for facilitating misuse index for explainable artificial intelligence in computing environments, according to one embodiment. A method of embodiments, as described herein, includes mapping training data with inference uses in a machine learning environment, where the training data is used for training a machine learning model. The
\end{abstract}


method may further include detecting, based on one or more policy/parameter thresholds, one or more discrepancies between the training data and the inference uses, classifying the one or more discrepancies as one or more misuses, and creating a misuse index listing the one or more misuses.

\title{
ANNEX 8
}

United States Patent Application

20190187775

Kind Code

Rotem; Nadav ; et al.

June 20, 2019

\section{DYNAMIC POWER MANAGEMENT FOR ARTIFICIAL INTELLIGENCE HARDWARE ACCELERATORS}

\begin{abstract}
A computer-implemented method for dynamically managing the power usage and/or performance of an artificial intelligence (AI) hardware accelerator may include (1) receiving an instruction stream that includes one or more instructions for performing at least one AIspecific computing task, (2) identifying a plurality of special-purpose, hardware-based functional units configured to perform AI-specific computing tasks, (3) predicting, based on an analysis of at least a portion of the instruction stream, a power-usage requirement for at least one of the functional units when executing the instruction stream, and then (4) modifying, based on the power-usage requirement, the power supplied to at least one of the functional units. Various other methods and systems are also disclosed.
\end{abstract}

\section{ANNEX 9}

United States Patent Application

20190188570

Kind Code 


\title{
METHODS AND APPARATUS FOR MODEL PARALLELISM IN ARTIFICIAL NEURAL NETWORKS
}

\begin{abstract}
The method according to an embodiment comprises automatically controlling allocation, to memories of available hardware resources, of parameters defining computational operations required to calculate an output of at least one layer of neurons of an artificial neural network. The allocation is controlled on the basis of previously-defined allocation data specifying how the operations required to calculate the output of the one layer of neurons are to be allocated to hardware resources to perform the operations. The allocation data is pre-defined using, at least partly, an automatic computer-implemented process, which may include checking before each iteration of the network which of the hardware resources are available to execute that iteration of the network and, if necessary, re-defining the allocation data for that iteration accordingly
\end{abstract}

\section{ANNEX 10}

United States Patent Application

20190192258

Kind Code

A1

KANG; Je Hun ; et al.

June 27, 2019

\section{AUTOMATIC STEPWISE TOOTH MOVEMENT SYSTEM AND METHOD USING ARTIFICIAL INTELLIGENCE TECHNOLOGY}

\begin{abstract}
Disclosed is a stepwise automatic orthodontic system and method using an artificial intelligence technique. The method includes: scanning a dental state of a patient by using an intraoral scanner; allowing a server to determine to which group of grouped data of the database the scanned dental data belong; allowing the server to refer to data of the determined group, move a tooth needing orthodontics gradually, and generate a predictive digital orthodontic dental data set; allowing the server to transmit the orthodontic-processed digital orthodontic dental data set of a patient to a 3D printer, and allowing the 3D printer to generate and output a dental orthodontic model; and generating a clear aligner by vacuum-compressing a transparent synthetic resin plate to the generated dental orthodontic model through a vacuum former. In the exemplary embodiment of the present invention, the orthodontic patient is clustered or grouped through an unsupervised learning based on the good orthodontic data
\end{abstract}


excluding personal information of the patient, and the tooth moving plan for orthodontics through repeated reinforcement learning satisfying the orthodontic limit condition suggested by the grouped data and the orthodontics textbook for respective steps.

\title{
ANNEX 11
}

\section{METHOD AND SYSTEM FOR CLASSIFYING AN OBJECT-OF-INTEREST USING AN ARTIFICIAL NEURAL NETWORK}

\begin{abstract}
Methods, systems, and techniques for classifying an object-of-interest using an artificial neural network, such as a convolutional neural network. An artificial neural network receives a sample image including the object-of-interest overlaying a background and a sample background image excluding the object-of-interest and corresponding to the background overlaid by the object-of-interest. The object-of-interest is classified using the artificial neural network. The artificial neural network classifies the object-of-interest using the sample background and sample images. Prior to receiving the sample background and sample images the artificial neural network has been trained to classify the object-of-interest using training image pairs. Each of at least some of the training image pairs includes a first training image that includes a training object-of-interest overlaying a training background and a training background image excluding the training object-of-interest and corresponding to the training background.
\end{abstract}

\section{ANNEX 12}

United States Patent Application

20190171950

Kind Code

Srivastava; Kumar

June 6, 2019 
METHOD AND SYSTEM FOR AUTO LEARNING, ARTIFICIAL INTELLIGENCE (AI) APPLICATIONS DEVELOPMENT, OPERATIONALIZATION AND EXECUTION

\begin{abstract}
Disclosed is a method and a system a method and a system for auto learning, artificial intelligence (AI) applications development, and execution. Various applications or operations may be associated with training environment-agnostic AI models, automated AI app application performance monitoring, fault, quality and performance remediation through prediction of failures or suboptimal performance, privacy and secure AI training and inference mechanism for data and AI model sharing between untrusted parties, and building auto learning applications that can automatically learn and improve.
\end{abstract}

\title{
ANNEX 13
}

United States Patent Application

20190171944

Kind Code

A1

LU; Mingzhu

June 6, 2019

\section{INTEGRITY EVALUATION OF UNSTRUCTURED PROCESSES USING ARTIFICIAL INTELLIGENCE (AI) TECHNIQUES}

\begin{abstract}
A process integrity evaluation system ensures integrity of unstructured processes. The process integrity evaluation system handles structured, semi-structured, and unstructured data at massive and large scale. The system provides scalability, secure storage, indexing, knowledge storage, and visualizations of processes by information retrieval, natural language processing, cloud computing, large scale machine learning, knowledge discovery, and other artificial intelligence techniques. Self-provided data, systematically gathered data, and potentially related data from additional sources are incorporated in the process integrity evaluation system which provides the core capabilities of data integrity checking, entity extraction, entity resolution, entity categorization, entity relationship extraction, processes extraction and reconstruction based on knowledge storage, such as knowledge graphs, inference functions, and evaluation computations. After extracting and reconstructing unstructured processes successfully, machine learning functions compute an integrity assurance score, e.g., a similarity, between extracted documents and the internal records in addition to an evaluation result, which can ensure the integrity of the unstructured processes.
\end{abstract}




\title{
ANNEX 14
}

United States Patent Application

20190140913

Kind Code

A1

GUIM BERNAT; Francesc ; et al.

May 9, 2019

TECHNIQUES FOR ARTIFICIAL INTELLIGENCE CAPABILITIES AT A NETWORK SWITCH

\begin{abstract}
Examples include techniques for artificial intelligence (AI) capabilities at a network switch. These examples include receiving a request to register a neural network for loading to an inference resource located at the network switch and loading the neural network based on information included in the request to support an AI service to be provided by users requesting the AI service.
\end{abstract}

\section{ANNEX 15}

United States Patent Application

20190171928

Kind Code

A1

Young; Robin

June 6, 2019

\section{DYNAMICALLY MANAGING ARTIFICIAL NEURAL NETWORKS}

\begin{abstract}
In some embodiments, the disclosed subject matter involves using socket layers with a plurality of artificial neural networks in a machine learning system to create customizable inputs and outputs for a machine learning service. The machine learning service may include a plurality of convolutional neural networks and a plurality of pre-trained fully connected neural networks to find the best fits. In an embodiment, when the customized input or output data is not a good fit with the pre-trained artificial neural networks, a socket layer may automatically request
\end{abstract}


additional convolutional layers or new training of a neural network to dynamically manage the machine learning system to accommodate the customized input or customized output. Other embodiments are described and claimed.

\title{
ANNEX 16
}

\section{SEARCH RESULT AGGREGATION METHOD AND APPARATUS BASED ON ARTIFICIAL INTELLIGENCE AND SEARCH ENGINE}

\begin{abstract}
The present disclosure provides a search result aggregation method and apparatus based on artificial intelligence and a search engine. The method includes: obtaining a query; generating a plurality of search results according to the query; obtaining a plurality of corresponding demand dimensions according to the query; aggregating the plurality of demand dimensions according to the plurality of search results; obtaining an answer corresponding to each demand dimension, and aggregating the answers corresponding to the plurality of demand dimensions according to the aggregated demand dimensions to generate an aggregation result.
\end{abstract}

\section{ANNEX 17}

United States Patent Application

Kind Code

Emma; Maria ; et al.
20190156222

A1

May 23, 2019 


\begin{abstract}
System, methods, and apparatuses for an artificial intelligence (AI) toy with improved conversational dialogue and personality development. The AI toy determines responses to stimuli based on user profiles and personality profiles that are developed through user interaction and external media inputs. Natural Language Processing (NLP) and other semantic interaction processing is paired with the profiles to develop AI personality and conversational ability.
\end{abstract}

